\title{
The MIPAS2D database of MIPAS/ENVISAT measurements retrieved with a multi-target 2-dimensional tomographic approach
}

\author{
B. M. Dinelli ${ }^{1}$, E. Arnone ${ }^{2}$, G. Brizzi ${ }^{2, *}$, M. Carlotti ${ }^{2}$, E. Castelli ${ }^{1,3}$, L. Magnani ${ }^{2}$, E. Papandrea ${ }^{2}$, M. Prevedelli ${ }^{2, * *}$, and \\ M. Ridolfi, ${ }^{\text {,** }}$ \\ ${ }^{1}$ ISAC-CNR - Istituto di Scienze dell'Atmosfera e del Clima, Via Gobetti, 101 - 40129 Bologna, Italy \\ ${ }^{2}$ Dipartimento di Chimica Fisica e Inorganica, Università di Bologna, Viale del Risorgimento, 4 - 40136 Bologna, Italy \\ ${ }^{3}$ IAC-CNR - Istituto per le Applicazioni del Calcolo "Mauro Picone", Via Madonna del Piano, 10 - 50019 Sesto \\ Fiorentino (FI), Italy \\ *now at: Serco SpA, Via Sciadonna, 24 - 00044 Frascati (RM), Italy \\ ** now at: Dipartimento di Fisica, Università di Bologna, Viale Berti Pichat, 6/2 - 40127 - Bologna, Italy
}

Received: 27 August 2009 - Published in Atmos. Meas. Tech. Discuss.: 16 October 2009

Revised: 5 March 2010 - Accepted: 9 March 2010 - Published: 17 March 2010

\begin{abstract}
We present a multi-year database of atmospheric fields of the upper troposphere, stratosphere and lower mesosphere retrieved from satellite measurements adopting a 2-dimensional tomographic approach. The full mission of the Michelson Interferometer for Passive Atmospheric Sounding (MIPAS) instrument, on board the European Space Agency ENVISAT satellite, is analyzed with the Geofit Multi-Target Retrieval (GMTR) system to obtain the MIPAS2D database with atmospheric fields of pressure, temperature and volume mixing ratio of MIPAS main targets $\mathrm{H}_{2} \mathrm{O}, \mathrm{O}_{3}, \mathrm{HNO}_{3}, \mathrm{CH}_{4}, \mathrm{~N}_{2} \mathrm{O}$, and $\mathrm{NO}_{2}$. The database covers both the MIPAS nominal observation mode measured at Full Resolution (FR) from July 2002 to March 2004 and the nominal observation mode of the new configuration, measured at Optimized Resolution (OR) and introduced in 2005. Further to the main targets, minor species $\mathrm{N}_{2} \mathrm{O}_{5}, \mathrm{ClONO}_{2}, \mathrm{COF}_{2}, \mathrm{CFC}-11$, and CFC-12 for the FR mission only have been included in MIPAS2D to enhance its applicability in studies of stratospheric chemistry. The database is continuously updated with the analysis of the ongoing measurements that are planned to last until the end of 2013 and extended to other targets. The GMTR algorithm is operated on a fixed vertical grid coincident with the tangent altitudes of the FR nominal mode, spanning the altitude range from 6 to $68 \mathrm{~km}$. In the horizontal domain, FR measurements are retrieved on both the observational
\end{abstract}

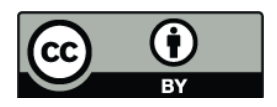

Correspondence to: B. M. Dinelli (bm.dinelli@isac.cnr.it) grid and an equispaced 5 latitudinal-degrees grid which is made possible by the 2-dimensional retrieval algorithm. The analysis of MIPAS OR observations is operated on the same altitude-latitude fixed retrieval grid used for the FR measurements. This choice provides a database with a homogeneous altitude and latitude grid, over the whole globe, covering to date about seven years of measurements. The equispaced latitude grid provides a new and convenient layout for the much needed synergetic studies of data from various instrumental and modeling sources. MIPAS2D is available to the scientific community through the two web sites http://www.mbf.fci.unibo.it/mipas2d.html, and http:// www.isac.cnr.it/ $\sim$ rss/mipas 2 d.htm.

\section{Introduction}

Four decades of Earth observation satellites have brought scientists an incomparable number of measurements, leading our way to a better understanding of the Earth's system and climate. Although our picture of the Earth's atmosphere has substantially improved over the years, there still remain a number of open issues that severely affect our knowledge of it. Among these issues are the behavior of the stratosphere and that of the upper troposphere-lower stratosphere region, and their influence on climate.

The global and multi-year coverage of satellite missions is giving us a detailed picture of physical and chemical quantities of the atmosphere, of their distribution, variability and long term trends. In this context limb sounding infrared

Published by Copernicus Publications on behalf of the European Geosciences Union. 
emission observations have the excellent characteristics of allowing global coverage in both day- and night-time (they do not require the presence of sunlight) with high vertical resolution.

Furthermore, the use of polar orbits permits full geographical coverage of the observations in a limited time interval to be obtained. However, not only is obtaining observations vital for understanding a changing atmosphere, but also the ability to properly extracting the relevant information from them. Although the short term variability and inhomogeneity of the atmosphere contain a signature of the processes governing the atmosphere, to date most of the online retrievals of remotely-sensed atmospheric measurements are still carried out with 1-dimensional algorithms assuming a horizontally homogeneous atmosphere (see e.g. Ridolfi et al., 2000; Raspollini et al., 2006; Kühl et al., 2008).

Satellite instruments scanning the atmospheric limb in the along-track direction permit to achieve a quite dense horizontal sampling of the atmosphere (with steps smaller than $500 \mathrm{~km}$ ), hence in principle permitting to investigate also the atmospheric horizontal variability. Examples of this type of instruments are the Michelson Interferometer for Passive Atmospheric Sounding (MIPAS) (Fischer et al., 2008) and the SCanning Imaging Absorption spectroMeter for Atmospheric CartograpHY (SCIAMACHY, http://www. sciamachy.org/) operating on board of the European Space Agency (ESA) ENVIronmental SATellite (ENVISAT), along with the Earth Observing System (EOS) Microwave Limb Sounder (MLS) instrument (Waters et al., 2006) on the Aura spacecraft.

State of the art 2-dimensional (2-D) tomographic retrieval algorithms (Carlotti et al., 2001; Steck et al., 2005; Livesey et al., 2006; Pukīte et al., 2008) make it possible to take into account a realistic inhomogeneity of the atmosphere in the along-track direction of limb-measurements and disclose more information from the measurements themselves. However, 2-D algorithms require a huge amount of computing resources in term of both computing time and memory allocation. To overcome the problem posed by the computing requirements, some retrieval codes (Stiller et al., 2002) while processing individual limb-scans include the possibility to model a horizontal temperature or Volume Mixing Ratio (VMR) gradient which, depending on the specific application, is either retrieved or assumed as known from external sources. Other 2-D retrieval codes, such as the one used for the routine analysis of the MLS measurements (Livesey et al., 2006), analyse a full orbit dividing it into "chunks" of subsequent limb scans and merge the results at the end of the retrieval process.

The 2-D Geofit Multi-Target Retrieval (GMTR) algorithm was developed by making use of several physical and computational optimizations (Carlotti et al., 2006) with the purpose of routinely analyzing the middle-infrared limb emission measurements acquired by the MIPAS instrument. Most of MIPAS measurements consist of rearward looking limb-scans with the instrument Line Of Sight (LOS) in the plane of ENVISAT's sun-synchronous nearly-polar orbit. Since there is overlap of the air masses sounded by the lines of sight of consecutive limb-scans, the GMTR algorithm exploits this cross-talk by combining in a simultaneous retrieval all the limb-scans of a complete orbit. This approach is implemented using a 2-D discretization of the atmosphere and thus enables the horizontal variability to be properly modelled.

We use the GMTR code to process routinely MIPAS measurements. The results are systematically archived into an easy-to-use database (MIPAS2D). The MIPAS2D database includes data covering the complete MIPAS mission for both the main targets selected for the ESA on-line retrievals and for a few additional minor species which showed the highest retrieval quality among the species detected by MIPAS. The target quantities included in the MIPAS2D database for the whole MIPAS mission are namely: pressure, temperature, and $\mathrm{VMR}$ of $\mathrm{H}_{2} \mathrm{O}, \mathrm{O}_{3}$, $\mathrm{HNO}_{3}, \mathrm{CH}_{4}, \mathrm{~N}_{2} \mathrm{O}, \mathrm{NO}_{2}$ (main targets), and for the period July 2002 to March 2004 it is complemented by the VMR of $\mathrm{N}_{2} \mathrm{O}_{5}, \mathrm{ClONO}_{2}, \mathrm{COF}_{2}, \mathrm{CFC}-11$, and CFC-12 (minor species). The extension of the analysis to further targets is under investigation. The MIPAS2D database covers the complete mission of the original MIPAS configuration (from July 2002 to March 2004), and it is continuously updated with measurements of the new configuration (since 2005 MIPAS optical path difference has been set to about $41 \%$ of the original one, leading to a degraded spectral resolution) currently planned to gradually stop after the end of 2013 . The MIPAS2D database is now available to the scientific community, and can be freely downloaded from the web.

The structure of this paper is the following: Sect. 2 describes the MIPAS instrument, the Level $1 \mathrm{~b}$ and the Level 2 data; Sect. 3 illustrates the main characteristics of the GMTR code and the selection of the retrieval grid; Sect. 4 describes the database along with the retrieval strategy; Sect. 5 contains examples of the possible use of the obtained results and finally conclusions are given in Sect. 6 .

\section{The MIPAS instrument}

MIPAS was developed by ESA as part of the payload of ENVISAT, successfully launched on 1 March 2002. MIPAS is a limb-scanning Fourier Transform (FT) spectrometer recording emission spectra in the mid-infrared, with a spectral range extending from 680 to $2410 \mathrm{~cm}^{-1}$. It was originally operated with a spectral resolution of $0.035 \mathrm{~cm}^{-1}$ full width half maximum, unapodised. ENVISAT achieves a global coverage of the Earth with 14.3 daily orbits with the descending (North to South) part crossing the equator at approximately 10:00 a.m. LT and the ascending part crossing it at approximately 10:00 p.m. LT. Most of MIPAS measurements during the Full Resolution (FR) mission 

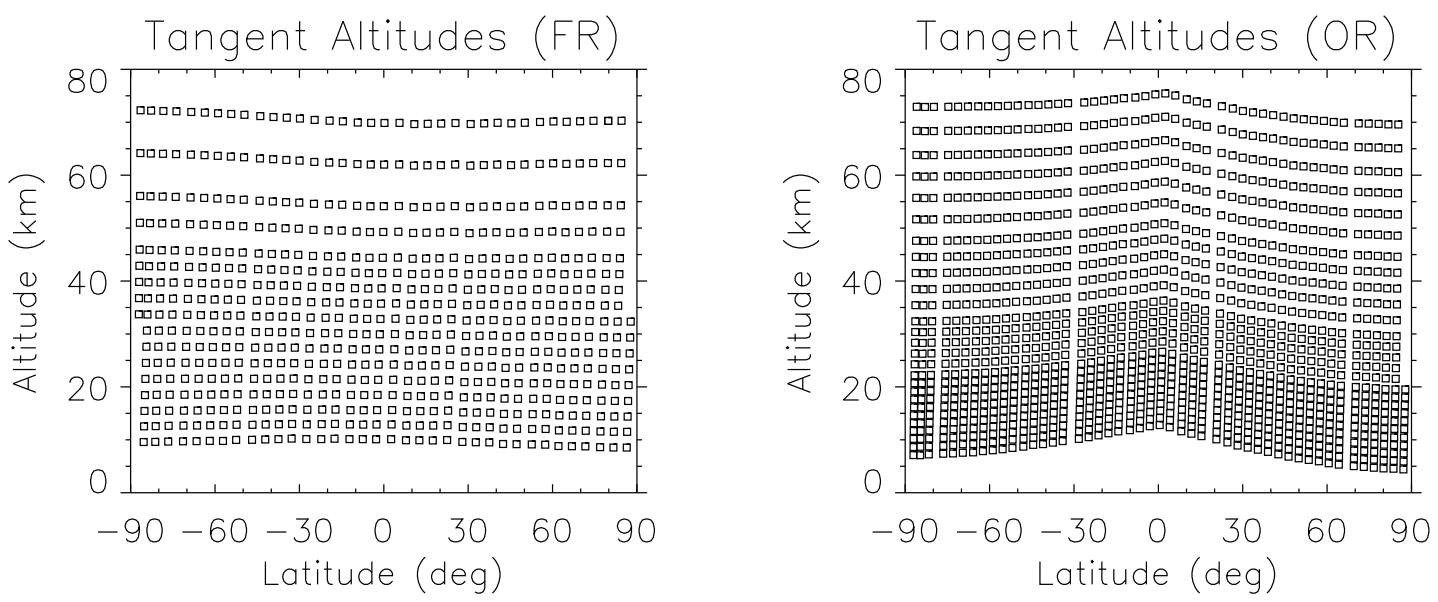

Fig. 1. Left: Tangent altitudes of the FR nominal mode vs. latitude. Right: the same but for the OR nominal mode.

(which lasted until March 2004) were taken in the nominal observation mode, with one orbit consisting of 72 limb-scans looking backward along the orbit track. Each of these limb-scans consists of 17 observations with tangent altitudes ranging from 6 to $68 \mathrm{~km}$, with $3 \mathrm{~km}$ steps up to $42 \mathrm{~km}$, and at 47, 52, 60 and $68 \mathrm{~km}$. MIPAS instantaneous field of view is approximately $3 \mathrm{~km}$ in height and $30 \mathrm{~km}$ across track at tangent point. In the FR nominal observation mode the average distance between tangent points of successive limb-scans is about $500 \mathrm{~km}\left(\sim 4.6^{\circ}\right.$ in latitude).

A minor part of MIPAS observations are acquired using the so called special observation modes, designed to enhance the instrument performance for a number of scientific objectives ranging from the study of the upper troposphere-lower stratosphere region to volcanic eruptions. These modes exploit different spectral resolutions, vertical scanning range and sampling steps, and can use sideways views.

Due to the deterioration of the interferometer slides, the FR mission was suspended on 26 March 2004. The technical problem was overcome in January 2005 by operating MIPAS with an Optimized Resolution (OR) at $41 \%$ of the maximum spectral resolution used in the original configuration. Since the reduced spectral resolution implied a reduced acquisition time of every limb view, all observation modes have been redefined increasing their spatial sampling. In the new OR nominal observation mode, each scan is made of $27 \mathrm{limb}$ views with tangent altitudes ranging from 3 to $70 \mathrm{~km}$ at variable altitude steps. Their nominal values are: 70.0, 66.0, $62.0,58.0,54.0,50.0,46.0,43.0,40.0,37.0,34.0,31.0$, $29.0,27.0,25.0,23.0,21.0,19.5,18.0,16.5,15.0,13.5$, $12.0,10.5,9.0,7.5,6.0 \mathrm{~km}$. While the vertical spacing between the tangent altitudes of the sweeps of the individual limb-scans is kept constant along the orbit, their offset is changed with latitude to follow the tropopause altitude (see Fig. 1 where the location of the tangent altitudes is reported as a function of the latitude for both FR and OR nominal modes). Although during the first two years of OR measurements the instrument availability was poor, since 15 December 2007 MIPAS is operated back at $100 \%$ of its duty cycle and is expected to perform well during the following years of the satellite life (until the end of 2013). The new duty cycle consists of 8 out of 10 days of nominal mode measurements plus 2 days of special observation modes: the pattern followed during the 10 days cycle has been changed several times since the restart of the full operations (see Oelhaf, 2008). A more detailed description of the MIPAS experiment can be found in Fischer et al. (2008).

\subsection{ESA MIPAS Level 1b data}

The first of the two main processing phases of the MIPAS mission ground segment produces ESA Level $1 b$ data. Level $1 \mathrm{~b}$ processing decodes the downlinked source packets of MIPAS interferograms transforming them into geolocated and calibrated atmospheric radiance spectra (Level $1 \mathrm{~b}$ spectra). The spectra are collected into Level $1 \mathrm{~b}$ files each containing the calibrated spectra acquired during a complete orbit. Details of the Level $1 \mathrm{~b}$ processing phases can be found in Kleinert et al. (2007) and references therein.

Level $1 \mathrm{~b}$ spectra are processed by ESA to obtain altitude profiles of atmospheric targets (Level 2 data). The same spectra are processed by our team using the GMTR to obtain the MIPAS2D database. In this section we recall some of the main features of Level $1 \mathrm{~b}$ data which affect the quality of MIPAS2D products and Level 2 products in general.

Level $1 \mathrm{~b}$ data for the FR mission were released by ESA and updated in successive improved processing versions. The current database of MIPAS Level $1 \mathrm{~b}$ data has been produced with different versions of the ESA Instrument Processing Facility (IPF): version 4.61 and 4.62 for the FR mission, and version 4.65 (up to 14 July 2006), and version 4.67 (afterwards) for the OR mission. 


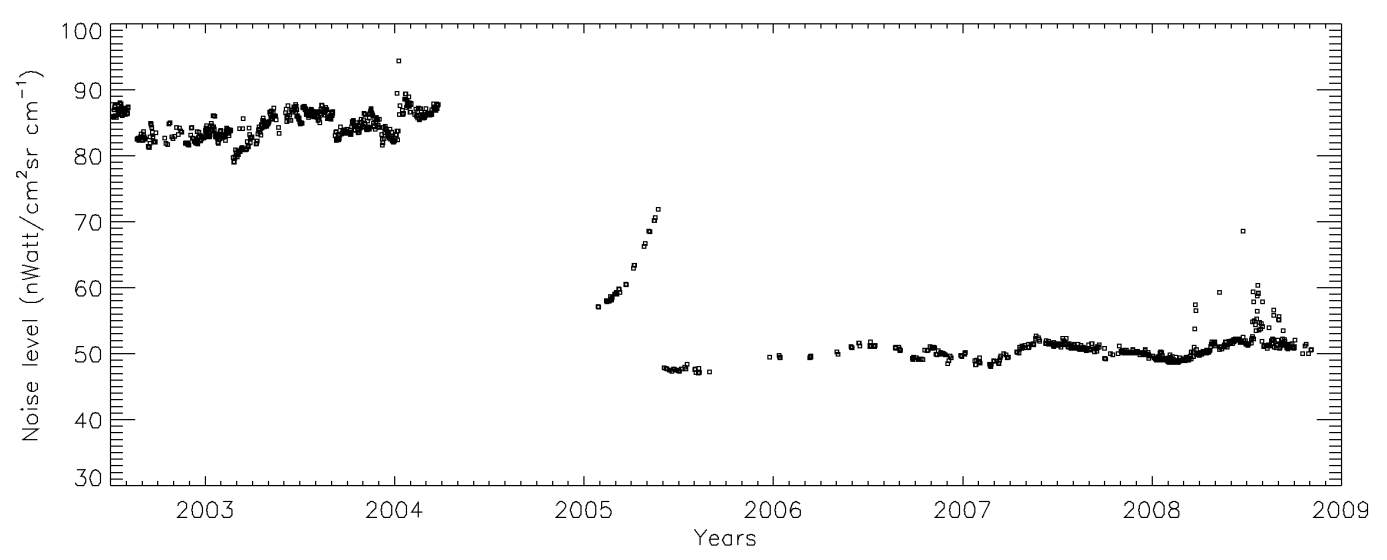

Fig. 2. MIPAS noise level for a $10 \mathrm{~cm}^{-1}$ wide spectral region centered at $690 \mathrm{~cm}^{-1}$ for the full MIPAS mission as a function of the acquisition time.

Figure 2 reports the noise level for a $10 \mathrm{~cm}^{-1}$ wide spectral region centered at $690 \mathrm{~cm}^{-1}$ for the full MIPAS mission. The figure shows that, as expected from radiometric considerations, the change in MIPAS spectral resolution (which occurred in January 2005) corresponds to a noise sharply reduced by a factor corresponding to the squared root of the ratio of the old to the new spectral resolution. Figure 2 also shows a discontinuity in the first part of the OR mission where the noise level first increases exponentially and then suddenly drops. This discontinuity was caused by the first ice decontamination process operated after the restart of MIPAS mission. The strong change in the noise level is due to the removal of the heavy ice layer accumulated on the detector windows during the long inactivity of the MIPAS instrument. The implications of this discontinuity in the results of the analysis of the full mission are discussed in Sects. 4.3.2 and 4.5.

Level $1 \mathrm{~b}$ spectra are reported on a frequency grid of $0.025 \mathrm{~cm}^{-1}$ for the FR mission and of $0.0625 \mathrm{~cm}^{-1}$ for the OR mission. The interferometric spectral resolution of the measurements, that is given by $1 / 2 L$ where $L$ is the maximum optical path difference of the interferometer $(20.016 \mathrm{~cm}$ and $8.2 \mathrm{~cm}$, respectively), is slightly different from these values. This implies that, to be reported on the above mentioned frequency grids, the spectra have to be resampled. Currently, MIPAS OR measurements processed with IPF versions 4.65 and 4.67 are affected by an error in the spectra-resampling procedure which is operated with an interpolating function that is not fully consistent with the FT spectral resolution. While awaiting for a new corrected ESA Level $1 \mathrm{~b}$ processor, we cope with this problem by emulating in the forward model the spectral distortions introduced by the resampling error. Tests performed running a GMTR analysis on a sample orbit processed with IPF 4.67 and with an error corrected prototype software, showed negligible differences (always laying within the retrieval error bars) therefore validating the approach we used for the OR measurements retrievals.
The selection process of the Level $1 \mathrm{~b}$ data used to generate the MIPAS2D database is discussed in detail in Sect. 4.2.

\subsection{ESA MIPAS Level 2 data}

Level 2 data of MIPAS main targets: pressure at tangent altitudes, temperature, and the VMRs of $\mathrm{H}_{2} \mathrm{O}, \mathrm{O}_{3}, \mathrm{HNO}_{3}$, $\mathrm{CH}_{4}, \mathrm{~N}_{2} \mathrm{O}$, and $\mathrm{NO}_{2}$, were continuously obtained from the nominal mode measurements in near real-time by ESA for the FR mission (Raspollini et al., 2006).

The change in the measurement strategy adopted for the OR mission required an upgrade of the Level 2 processor, and to date just 140 orbits of the OR mission have been analysed by ESA with the prototype version of the new Level 2 code for validation purposes. However, independent systematic analyses of MIPAS data (FR and OR missions) have been performed by Oxford University (Dudhia et al., 2007) and FZK/IMK (see von Clarmann et al., 2009a, and references therein) with their scientific processors.

We briefly recall the main features of ESA Level 2 data to appreciate the differences with the retrieval strategy adopted in the MIPAS2D database. ESA Level 2 data for the FR mission have been obtained using the Optimized Retrieval Model (ORM, Ridolfi et al., 2000). ORM implements the Global-fit (Carlotti, 1988) algorithm by using several approximations to meet the very demanding runtime requirements of the near real-time processing. For each target, the retrieval is performed on a set of small spectral regions called Micro Windows (MWs), selected in order to have the best retrieval accuracy of the final Level 2 products (Dudhia et al., 2002). All main targets are retrieved in cascade, using the results of previously retrieved targets in the subsequent steps of the retrieval chain, and following a sequence designed to minimize the error propagation. An off-line retrieval is also performed by ESA with the same Level 2 processor on more consolidated Level $1 \mathrm{~b}$ data using the best available correlative data, therefore improving the quality of ESA Level 2 off-line products. 
ESA Level 2 products, derived with the described retrieval strategy, are based on three main assumptions:

1. ORM assumes a horizontally homogeneous atmosphere. This assumption can significantly affect the error budget of the retrieved quantities, since the horizontal homogeneity assumption can fail in presence of significant horizontal structures along the instrument LOS.

2. Within the analysis of each limb-scan, ORM performs a sequential retrieval of the targets. Although optimized, the sequential retrieval leads to a propagation of the retrieval error on successively retrieved targets by effect of the reciprocal contamination in the analyzed spectral features.

3. MIPAS limb-scans are individually analysed by ORM, using the geolocation of the tangent points to define the retrieval grid. As a consequence, the retrieved profiles are calculated on a sparse horizontal and vertical grid, and the horizontal resolution is implicitly defined by the separation between adjacent scans (see von Clarmann et al., 2009b).

The approximations described at points 1 . and 2. affect significantly the error budget of the retrieved quantities. The impact of these systematic errors is limited with a careful selection of the spectral points of each MW to be included in the analyses. However, under certain circumstances, the residual errors are significant (see for instance Arnone et al., 2009a).

The aforementioned independent analyses of MIPAS observations performed at Oxford (Dudhia et al., 2007) and IMK (von Clarmann et al., 2003; Milz et al., 2005) are based on similar assumptions; in particular they retrieve all targets analysing individual limb-scans on the horizontal grid defined by the geolocation of MIPAS scans and assuming the atmosphere horizontally homogeneous. Therefore the obtained products are affected by approximations similar to those of the ESA Level 2 analysis. Recently von Clarmann et al. (2009a) have introduced in the retrievals of the OR observations a horizontal temperature gradient (applied only in the atmospheric region close to the tangent points) to cope with the horizontal inhomogeneities of the sampled atmosphere in order to improve the convergence rate.

\section{The 2-dimensional geofit multi-target retrieval (GMTR) algorithm}

As a response to the shortcomings highlighted in the previous section, Carlotti et al. (2006) developed the GMTR 2-D algorithm. The GMTR retrieval system is an open source package, specifically designed for MIPAS measurements, developed under an ESA contract and delivered to ESA for inclusion in the BEAT tools repository (http://envisat.esa. int/services/beat). GMTR analyses of the special modes of the FR mission (along with the analysis of few orbits for the nominal observation mode) have been validated by Ceccherini and Mencaraglia (2004) during the ESA study that lead to the development of the code. An upgraded version of GMTR that, among the novel features, is able to handle the Level $1 \mathrm{~b}$ data of MIPAS OR configuration, was used to generate the MIPAS2D database. The most important features of the GMTR algorithm are briefly summarized below. A detailed description of the analysis system can be found in Carlotti et al. (2006).

The GMTR is based on the Geo-fit approach (Carlotti et al., 2001) upgraded with the Multi-Target Retrieval (MTR) functionality (Dinelli et al., 2004). The Geo-fit performs a tomographic retrieval on observations collected along a whole orbit operating a 2-D discretization of the atmosphere which enables the horizontal atmospheric structures to be modelled. In the Geo-fit approach each limb observation contributes to determining the unknown quantity at a number of different locations among those spanned by its line of sight. MIPAS nominal mode measurement strategy, with the LOS lying approximately in the orbit plane, enables the gathering of information about a given location of the atmosphere from all the lines of sight that cross that location regardless of the scan they belong to. The MTR approach enables to perform simultaneous fit of different targets thus eliminating the systematic error components due to the propagation of the uncertainties in pressure, temperature and in the concentrations of molecules that generate interfering spectral features on the species included in the simultaneous retrieval.

The GMTR version used to generate the MIPAS2D database also includes the possibility of performing the retrievals using the Optimal Estimation (OE) or maximum a posteriori approach (Rodgers, 2000). This new functionality has been used for the database generation to preserve the possibility of adopting a regular retrieval grid also in the case of a lack of observations and of the variable altitude offset of the OR limb scans along the orbit. To limit the size of the matrices involved in the inversion processes only a diagonal Variance-Covariance Matrix (VCM) of the a-priori information has been used in the analyses.

\subsection{MIPAS2D retrieval grids}

The Geo-fit is implemented in GMTR by defining a 2-D retrieval grid described by a vertical coordinate (spanning the altitude range of the observations) and an angular coordinate (following the satellite position along the orbit) called Orbital Coordinate (OC) and performing the radiative transfer through a 2-D discretized atmosphere. Since GMTR simultaneously fits observations from a complete orbit that originates and terminates at the North Pole, the OC spans from $0^{\circ}$ at the North Pole, through $180^{\circ}$ at the South Pole up to $360^{\circ}$ back to the North Pole. The fact that the 


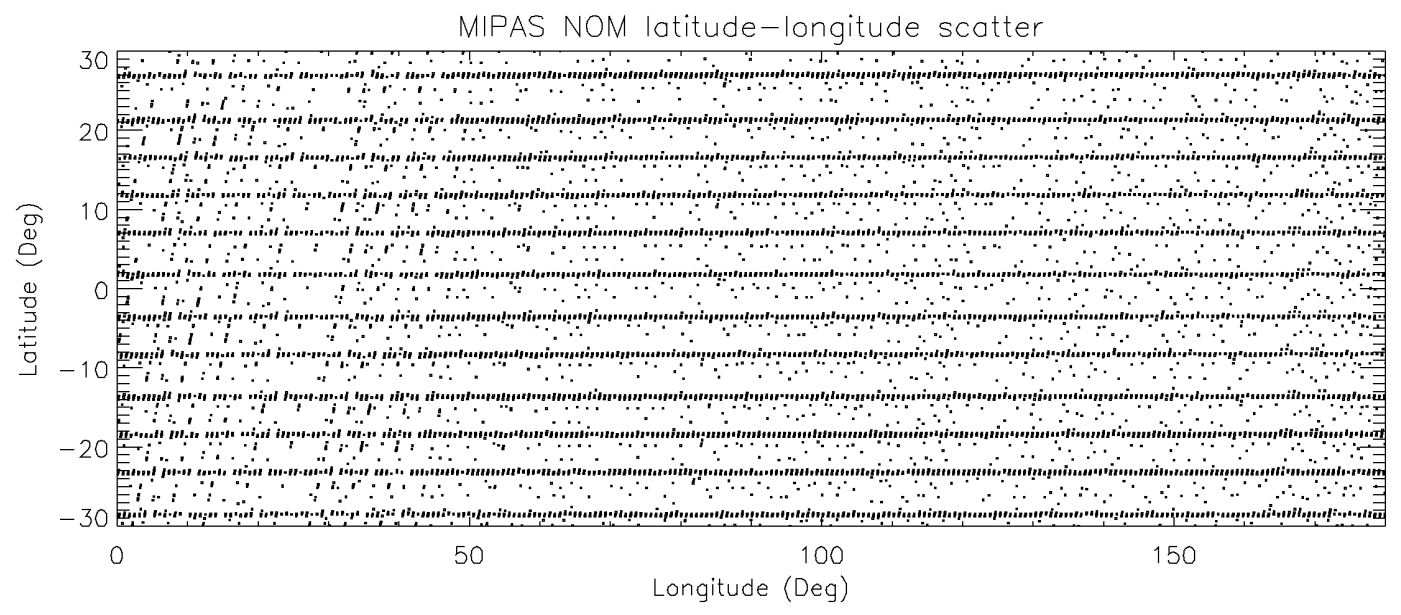

Fig. 3. Geolocation of the retrieved NOM profiles (latitude versus longitude) in the equatorial region for the complete FR mission.

observations of a whole orbit are simultaneously analysed makes it possible to define a retrieval grid that is totally independent from the measurements' geolocation. This is an advantage with respect to the 1-dimensional retrievals that can also determine atmospheric profiles on custom made vertical grids, but are tied to the position of the limb-scans for the horizontal location of the retrieved profiles.

As already stated in Sect. 2 the geolocation of the tangent points of MIPAS measurements for both FR and OR missions are not regularly spaced in the vertical and horizontal domains. In the vertical domain each scan has its own tangent altitudes different from the commanded ones even in the FR measurements. These differences arise from a combination of several effects among which unaccounted variations of satellite pitch and the different refractive indices of the inhomogeneous atmosphere sampled by the lines of sight. Moreover, the motion of the satellite during the acquisition time of each limb view is only partially compensated by the measurement strategy and the tangent points of each scan are not vertically aligned. If the 2-D retrieval grid were defined at the tangent points, the spread of the tangent altitudes would lead to a complicated interpolation process for both the construction of the atmospheric fields on the 2-D discretization of the atmosphere and the representation of the results. The 2-D interpolation processes can be easily performed if one of the 2-D grid dimensions (vertical or horizontal) is fixed over the whole orbit. For this reason we have adopted a vertical retrieval grid located at fixed altitudes along the whole orbit, corresponding to the vertical grid of the nominal mode of the FR mission, i.e. from 6 to $68 \mathrm{~km}$ with a $3 \mathrm{~km}$ step from 6 to $42 \mathrm{~km}$ and then at $47,52,60$ and $68 \mathrm{~km}$. The same vertical grid has been used for both FR and OR missions in order to obtain a geometrical homogeneity of the database.

As a consequence of this choice, the horizontal retrieval grid can be defined at arbitrary positions and it is not forced to coincide with the geolocation of the measurements.
A natural choice for the horizontal grid would be to use the OC of the tangent points of the measurements. However, since the tangent points of individual limb-views of the same scan are not vertically aligned, in order to ease the interpretation of the retrieval results we set the retrieval grid points vertically aligned at selected values of the OC corresponding to the average orbital coordinates of the tangent points of each limb-scan. By convention, we have labelled "NOM" the dataset of MIPAS2D products obtained with this horizontal grid. The NOM grid follows the horizontal sampling of MIPAS measurements. Figure 3 shows the geolocation (latitude versus longitude) of all retrieved profiles for the NOM grid of the FR mission in the equatorial region (descending part of the orbit only). Although the latitude of the NOM profiles tends to cluster around preferred locations, due to the synchronization of the scan pattern to start at a particular latitude performed every orbit, a large fraction of profiles does not, therefore requiring horizontal interpolation even just to obtain a latitude-consistent database of the available measurements. Moreover the distance between these preferred latitudes varies between about $4.6^{\circ}$ and $5.3^{\circ}$ along the orbit, so that all NOM measurements require a horizontal interpolation if they have to be reported on a constant latitude grid (as used by some atmospheric models). To minimize the need of interpolation in averaging processes and to ease the intercomparison with data from other instruments or atmospheric models, the best horizontal grid is a fixed layout of equispaced OCs for all the analyzed orbits. We label "GRD" the dataset corresponding to this fixed horizontal grid. The GRD retrieval grid originates at the North Pole and extends along the full orbit with profiles at OCs (and thus latitudes) equispaced by $5^{\circ}$. Both horizontal grids have been applied to the FR measurements.

The OR nominal mode samples the atmosphere with finer vertical and horizontal steps with respect to the FR nominal mode. Moreover the tangent points of the measurements 
span an altitude range that is latitude dependent (see Fig. 1). The spread of the tangent altitudes in both vertical and horizontal domains makes the GMTR analysis on a NOM grid less meaningful; furthermore the exploitation of the finer vertical and horizontal grid makes the retrieval unstable and therefore would require the use of an off-diagonal regularization scheme, making the results of the analysis not directly comparable with the FR results. For this reason for the first release of the database the analysis of the OR mission has been performed only on the GRD grid.

\section{The MIPAS2D database}

In this Section we summarize the retrieval strategy, the selection of the observations used for the analyses, the sensitivity of the results to the adopted parameters and the filtering applied to obtain the released MIPAS2D version 1.0.

\subsection{Retrieval strategy}

For the generation of MIPAS2D, GMTR was configured to obtain a spatially homogeneous database along the two parts of the mission and to facilitate the use of MIPAS2D data with atmospheric models and other observational datasets.

All the main targets are retrieved for both the FR and OR missions on the GRD retrieval grid. As discussed in Dinelli et al. (2004), because of their correlations, pressure, temperature, water vapor and ozone are jointly retrieved by exploiting the MTR functionality of the code. All the other species are then retrieved sequentially assuming the previously retrieved atmospheric fields. The adopted sequence: $\mathrm{HNO}_{3}, \mathrm{CH}_{4}, \mathrm{~N}_{2} \mathrm{O}$, and $\mathrm{NO}_{2}$, that is the same used by ESA in the operational retrievals, was designed to minimize the systematic error deriving from the non-correct modeling of the previously retrieved species. For the FR mission the additional NOM database was produced, also including the minor species $\mathrm{N}_{2} \mathrm{O}_{5}, \mathrm{ClONO}_{2}, \mathrm{COF}_{2}$, CFC-11, and CFC-12 that are retrieved individually in this order after the main targets. The 2-D fields of all targets were retrieved simultaneously with the 2-D field of the absorption coefficients (one for each MW) of the atmospheric continuum up to the altitude of $25 \mathrm{~km}$. The vertical range on which the retrieval was performed was optimized for individual atmospheric targets according to their altitude distribution: while pressure, temperature, $\mathrm{H}_{2} \mathrm{O}$, $\mathrm{O}_{3}, \mathrm{CH}_{4}$, and $\mathrm{N}_{2} \mathrm{O}$ have been retrieved on the full vertical range (i.e. from 6 to $68 \mathrm{~km}$ ), $\mathrm{HNO}_{3}, \mathrm{NO}_{2}$, and the additional targets, have been retrieved on the vertical range where total errors below $100 \%$ are predicted (see Sect. 4.3.1). The retrievals are performed using the $\mathrm{OE}$ technique in order to avoid singularities in the inversion procedure caused by the presence of atmospheric regions not sampled by the observations included in the retrieval because of e.g., measurement strategy, corrupted sweeps or presence of
Table 1. Size of the a-priori errors used in the retrievals.

\begin{tabular}{lll}
\hline Target & Constant error & Fractional error \\
\hline Pressure & - & $30 \%$ \\
Temperature & $7 \mathrm{~K}$ & - \\
$\mathrm{H}_{2} \mathrm{O}$ & $1 \mathrm{ppmV}$ & $80 \%$ \\
$\mathrm{O}_{3}$ & $0.5 \mathrm{ppmV}$ & $80 \%$ \\
$\mathrm{HNO}_{3}$ & $0.5 \mathrm{ppbV}$ & $80 \%$ \\
$\mathrm{CH}_{4}$ & $0.01 \mathrm{ppmV}$ & $80 \%$ \\
$\mathrm{~N}_{2} \mathrm{O}$ & $0.05 \mathrm{ppmV}$ & $80 \%$ \\
$\mathrm{NO}_{2}$ & $0.01 \mathrm{ppmV}$ & $10 \%$ \\
$\mathrm{~N}_{2} \mathrm{O}_{5}$ & $0.2 \mathrm{ppbV}$ & $80 \%$ \\
$\mathrm{ClONO}_{2}$ & $0.2 \mathrm{ppbV}$ & $80 \%$ \\
$\mathrm{COF}_{2}$ & $0.1 \mathrm{ppbV}$ & $80 \%$ \\
$\mathrm{CFC}-11$ & $0.1 \mathrm{ppbV}$ & $80 \%$ \\
$\mathrm{CFC}-12$ & $0.2 \mathrm{ppbV}$ & $80 \%$ \\
\hline
\end{tabular}

clouds in the LOS. With OE the a-priori information and the associated a-priori errors are an important choice because they can affect the quality of the obtained products. In the analyses we have adopted the strategy to use the a-priori information also as initial guess of the retrievals. The choice of the initial atmospheric status is discussed in Sect. 4.2.2. Tests have been performed to tune the size of the a-priori errors in order to avoid biases due to the chosen a-priori information (initial guess). The a-priori errors have been defined as the aritmetic sum of a fraction of the value of the a-priori plus a constant term introduced to prevent strong constraints where the a-priori fields assume very small values. Table 1 shows the values used for all the considered targets. As shown in this table, for all the molecules but $\mathrm{NO}_{2}$ the percent part of the a-priori error was set to $80 \%$. For $\mathrm{NO}_{2}$, since a high variability is expected due to either its day-night variation or to the high altitude variability of its VMR, we have chosen to use a large value for the constant part and a smaller percent part in the whole altitude range. The a-priori error of pressure was tuned to $30 \%$ in order to account for MIPAS pointing errors. For the continuum absorption coefficients, a very large a-priori error has been assumed about $10^{6}$ larger than the a-priori value in order to avoid constraints on the retrieved values.

For technical reasons the GMTR analysis system does not allow for the retrieved parameters to assume negative values: if during the retrieval iterations the correction to be applied to one parameter leads to a negative value, the parameter is arbitrarily set to $1.0 \mathrm{E}-14$.

The spectra affected by the presence of clouds in the LOS are filtered out using the method proposed by Spang et al. (2004) adopting a cloud index (CI) threshold equal to 4.0. This conservative value prevents the use of spectra even partially contaminated by clouds.

The sensitivity of the database products to the adopted retrieval configuration is described in Sect. 4.3. 


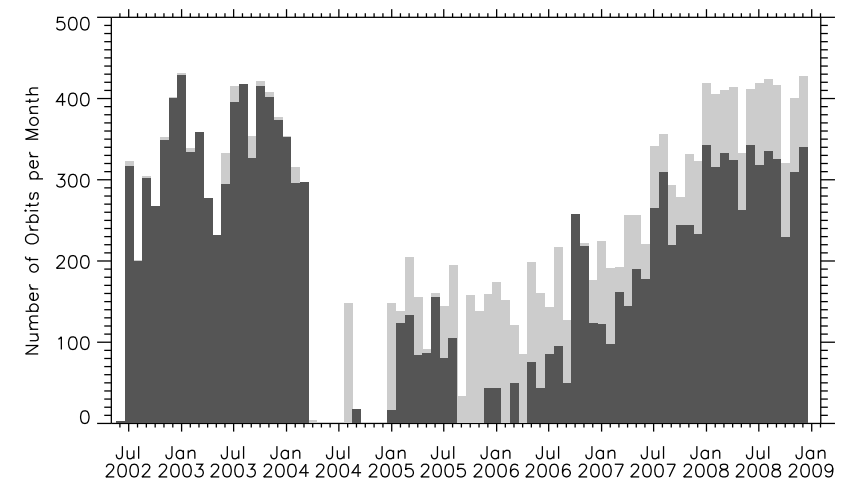

Fig. 4. Distribution of the number of Level $1 \mathrm{~b}$ orbits per month processed in the MIPAS2D (black), and of the total number of available Level $1 \mathrm{~b}$ orbits (gray). Note that the total number of Level $1 \mathrm{~b}$ orbits include also the special observation modes.

\subsection{Input data}

\subsubsection{Level $1 \mathrm{~b}$ data selection}

Since ENVISAT is on a nearly-polar orbit, the atmospheric regions where the geographical distance between the air masses observed by consecutive orbits is minimum are at the Earth's Poles. Therefore one of the two Poles is the best choice to originate the analysed orbit. For the MIPAS2D database, as discussed in Sect. 3, the North Pole identifies the starting and ending point of the analysed orbits. However the first limb-scan reported in ESA Level $1 \mathrm{~b}$ files is always at latitudes far from $90^{\circ} \mathrm{N}$; therefore in order to obtain a complete orbit to be analysed with GMTR the measurements contained in the Level $1 b$ files of two consecutive orbits are merged in a single analysis (see Carlotti et al., 2006). Whenever consecutive orbits are not available, the retrieval is performed on the measurements acquired after reaching the North Pole of a single Level $1 \mathrm{~b}$ file without closure of the geographical loop.

A selection of the Level $1 \mathrm{~b}$ files has been necessary because of the several Level 1 reprocessings performed by ESA and leading to the release of non-unique Level $1 \mathrm{~b}$ files for each orbit over time. The selection has been performed adopting criteria based on the geographical coverage of the files and on quality indicators. Whenever multiple Level $1 \mathrm{~b}$ files exist for the same orbit we retained the one processed with the latest version of the IPF spanning the wider geographical region. If a further selection is needed, the most recent released file is retained unless evident anomalies are found during an automated and manual check. An ad-hoc list of Level $1 \mathrm{~b}$ data was then generated with a unique file for each orbit. Figure 4 shows in black the histogram of the number of Level $1 \mathrm{~b}$ orbits per month analysed to produce the MIPAS2D database. The figure also reports in gray the total number of available orbits per month, including the orbits measured in the special observation modes, that have not been included in the current analysis. Tests performed to see if the different IPF versions used to produce the Level $1 \mathrm{~b}$ data were producing inhomogeneities in the retrieval results showed no significant effect.

\subsubsection{Auxiliary data}

As mentioned in Sect. 4.1 we exploit the $\mathrm{OE}$ method for the retrieval of our Level 2 products and the a-priori information is provided by the same atmospheric fields used as initial guess for the Gauss-Newton iterative procedure. As discussed in Sect. 4.1, the size of the a-priori errors is such that the result of the retrieval is independent from the initial guess used for the target quantity, provided that the analysed observations contain sufficient information (see Sect. 4.3). However, an initial guess close to the real fields reduces the number of Gauss-Newton iterations needed to reach a stable result, thus limiting the computing time. Therefore, whenever available, ESA Level 2 data were used as initial guess in the analysis of the FR mission. As for the OR mission, where ESA Level 2 data are not yet available, we have used the results obtained for the FR mission for a good-quality orbit of the corresponding month of 2003. Only for a very limited number of cases, where this strategy was not possible, the initial guess profiles were taken from the climatological IG2 database (Remedios et al., 2007). The same database has been used to complement the initial guess profiles outside the vertical retrieval range and to define the atmospheric state relative to all the non-target molecules of the retrieval procedure. The boundary of the atmosphere was set to $80 \mathrm{~km}$.

For each target (or group of targets in the case of MTR) the analysis has been performed using dedicated MWs (different for the FR and OR mission) selected with the algorithm MWMAKE, described in Dudhia et al. (2002) (http://www. atm.ox.ac.uk/RFM/mwmake.html). The frequency intervals of the used MWs are reported in Table 2. The MWs used for the joint retrieval of pressure, temperature, $\mathrm{H}_{2} \mathrm{O}$ and $\mathrm{O}_{3}$ VMRs, as well as those used for the retrieval of the minor species not included in the ESA operational analysis, have been generated for a 2-D analysis that exploits the MTR functionality. For $\mathrm{HNO}_{3}, \mathrm{CH}_{4}, \mathrm{~N}_{2} \mathrm{O}$ and $\mathrm{NO}_{2}$ we have chosen to use the MWs of the ESA Level 2 ground processor. The possibility of using dedicated MWs, selected for a 2-D analysis, is under investigation for future releases of the database.

Auxiliary data for the GMTR analysis system are also the Occupation Matrices (OM) that define the altitude range where each MW is used in the retrievals and the altitude dependent "spectral masks" used to exclude specific spectral points from the inversion for a reduction of the size of the systematic errors affecting the retrievals. All these auxiliary data have been generated with the MWMAKE program. Spectroscopic data are not directly handled by the GMTR retrieval system, since the absorption cross sections of each 
Table 2. List of the MWs $\left(\mathrm{cm}^{-1}\right)$ used for the retrievals of both FR and OR measurements. Note that specific spectral points within the MWs might be excluded from the retrieval to minimize the total errors.

\begin{tabular}{|c|c|c|c|c|}
\hline \multicolumn{5}{|c|}{ FR analysis } \\
\hline MTR & $\mathrm{HNO}_{3}$ & $\mathrm{CH}_{4}$ & $\mathrm{~N}_{2} \mathrm{O}$ & $\mathrm{NO}_{2}$ \\
\hline $\begin{array}{r}685.200-686.200 \\
713.200-714.200 \\
757.950-758.950 \\
767.975-768.975 \\
1034.150-1035.150 \\
1393.475-1394.475 \\
1616.500-1617.500\end{array}$ & $\begin{array}{l}876.375-879.375 \\
885.100-888.100\end{array}$ & $\begin{array}{l}1227.175-1230.175 \\
1350.875-1353.875\end{array}$ & $\begin{array}{l}1233.275-1236.275 \\
1272.050-1275.050\end{array}$ & $\begin{array}{l}1607.275-1610.275 \\
1613.725-1616.600 \\
1622.550-1623.475\end{array}$ \\
\hline \multicolumn{5}{|c|}{ OR analysis } \\
\hline $\begin{array}{r}686.688-689.688 \\
689.750-692.750 \\
731.188-734.188 \\
790.625-793.625 \\
1036.312-1039.312 \\
1071.875-1074.875 \\
1651.000-1654.000 \\
1682.688-1685.688\end{array}$ & $\begin{array}{l}836.875-839.875 \\
859.562-862.562 \\
877.000-880.000 \\
893.625-896.625 \\
918.938-921.938\end{array}$ & $\begin{array}{l}1219.062-1222.062 \\
1228.438-1231.438 \\
1233.812-1236.812 \\
1281.562-1284.562 \\
1304.562-1307.562\end{array}$ & $\begin{array}{l}1230.375-1233.312 \\
1233.562-1235.812 \\
1256.688-1259.562 \\
1271.625-1274.625 \\
1276.312-1279.312\end{array}$ & $\begin{array}{l}1570.500-1573.500 \\
1602.500-1605.500 \\
1608.625-1611.625 \\
1613.500-1616.500 \\
1626.000-1629.000\end{array}$ \\
\hline \multicolumn{5}{|c|}{ Minor Species FR analysis } \\
\hline $\mathrm{N}_{2} \mathrm{O}_{5}$ & $\mathrm{ClONO}_{2}$ & $\mathrm{COF}_{2}$ & CFC-11 & CFC-12 \\
\hline $\begin{array}{r}742.900-745.900 \\
746.100-749.100 \\
750.650-753.350 \\
1238.825-1241.775 \\
1245.675-1248.675\end{array}$ & $\begin{array}{r}778.525-781.525 \\
805.400-808.400 \\
808.425-811.425 \\
1282.125-1285.000 \\
1285.025-1286.550 \\
1290.625-1293.625 \\
1303.350-1306.350\end{array}$ & $\begin{array}{r}772.000-775.000 \\
784.425-785.275 \\
1223.925-1225.100 \\
1225.750-1228.750 \\
1228.900-1230.275 \\
1230.325-1231.325 \\
1236.450-1237.250 \\
1252.600-1253.250 \\
1253.350-1255.575 \\
1256.625-1259.625\end{array}$ & $\begin{array}{l}839.625-842.625 \\
842.650-845.650 \\
846.450-849.450 \\
849.475-852.475 \\
853.075-856.075 \\
856.250-859.250\end{array}$ & $\begin{array}{r}885.975-888.975 \\
918.375-921.375 \\
921.400-924.400 \\
924.425-927.425 \\
929.125-932.125 \\
1159.700-1162.700\end{array}$ \\
\hline
\end{tabular}

gas are derived from precalculated look-up tables. The spectroscopic data used to compute them are taken from the MIPAS dedicated spectroscopic database (Flaud et al., 2003). Further auxiliary input data were taken from the GMTR package (see Carlotti et al., 2006).

\subsection{Data quality and parameter sensitivity}

The strategies adopted for the selection of the retrieved parameters and for the setup of the analysis environment were optimized with a series of dedicated test retrievals. In particular, an extended test was performed over all MIPAS measurements acquired from 1 to 20 October 2003 in order to assess the sensitivity of the results to the initial guess profiles and to the size of the errors assigned to the a-priori information.

We report the results of these activities in the following subsections where we describe the size of the systematic errors affecting the Level 2 products included in the database
(Sect. 4.3.1), the quality assessment of these products (Sect. 4.3.2), the behavior of the retrieval procedure when single orbits or orbit segments are analysed (Sect. 4.3.3), the sensitivity to pointing calibrations (Sect. 4.3.4) and the difference between the NOM and the GRD datasets (Sect. 4.3.5).

\subsubsection{Systematic errors}

The algorithm used to select the MWs (Dudhia et al., 2002) enables an a-priori assessment of the systematic errors affecting the final products of the analysis. These errors are assumed to be common to all the analyses that use the same set of MWs, they are characteristic of each target and change for the FR and the OR Level 2 products. The systematic errors whose effects have been quantified are:

- errors due to the assumptions used in the forward model internal to the retrieval system (i.e. local thermal 
Table 3. Systematic errors affecting the retrieved values. The errors are all given in $\%$ apart from temperature errors whose unit is K. Only values for which the estimated random error is below $100 \%$ are reported in the table.

\begin{tabular}{|c|c|c|c|c|c|c|c|c|c|c|c|c|c|c|c|c|c|}
\hline \multicolumn{18}{|c|}{ FR analysis } \\
\hline Alt $[\mathrm{km}]$ & 6.0 & 9.0 & 12.0 & 15.0 & 18.0 & 21.0 & 24.0 & 27.0 & 30.0 & 33.0 & 36.0 & 39.0 & 42.0 & 47.0 & 52.0 & 60.0 & 68.0 \\
\hline pres. & 3.8 & 3.6 & 2.8 & 2.6 & 2.5 & 2.3 & 2.2 & 2.1 & 2.0 & 1.9 & 2.1 & 2.4 & 2.5 & 2.7 & 3.1 & 3.3 & 3.5 \\
\hline temp. & 1.35 & 0.68 & 0.45 & 0.49 & 0.54 & 0.50 & 0.56 & 0.47 & 0.49 & 0.51 & 0.71 & 0.90 & 1.04 & 1.07 & 1.49 & 2.26 & 5.06 \\
\hline $\mathrm{H}_{2} \mathrm{O}$ & 12.1 & 8.9 & 16.4 & 12.5 & 12.6 & 8.0 & 9.3 & 9.8 & 10.2 & 9.7 & 12.4 & 12.0 & 15.7 & 13.4 & 16.5 & 66.9 & - \\
\hline $\mathrm{O}_{3}$ & 20.3 & 14.6 & 11.7 & 14.2 & 13.1 & 4.1 & 4.6 & 4.6 & 4.8 & 6.3 & 5.5 & 5.4 & 8.0 & 6.2 & 6.1 & 10.8 & 42.9 \\
\hline $\mathrm{HNO}_{3}$ & - & 21.4 & 17.9 & 10.2 & 7.3 & 6.2 & 6.1 & 7.1 & 8.0 & 9.7 & 9.7 & 11.0 & 22.2 & - & - & - & - \\
\hline $\mathrm{CH}_{4}$ & 14.4 & 8.5 & 10.6 & 11.4 & 12.9 & 12.7 & 12.7 & 11.7 & 10.7 & 11.4 & 9.0 & 9.0 & 9.3 & 9.1 & 10.9 & 29.0 & 38.4 \\
\hline $\mathrm{N}_{2} \mathrm{O}$ & 8.1 & 5.0 & 9.1 & 12.2 & 10.2 & 10.1 & 10.8 & 12.6 & 18.9 & 13.7 & 15.7 & 10.6 & 11.1 & 25.0 & 86.6 & - & - \\
\hline $\mathrm{NO}_{2}$ & - & - & - & - & - & - & 14.3 & 11.3 & 8.0 & 7.3 & 6.6 & 5.7 & 8.7 & 19.0 & 24.5 & 37.8 & 46.8 \\
\hline $\mathrm{N}_{2} \mathrm{O}_{5}$ & - & - & - & 34.4 & 28.0 & 22.3 & 16.1 & 12.8 & 7.4 & 8.5 & 8.7 & 17.5 & 14.1 & - & - & - & - \\
\hline $\mathrm{ClONO}_{2}$ & - & 21.8 & 29.9 & 16.9 & 7.6 & 7.2 & 5.3 & 7.0 & 3.6 & 6.1 & 7.6 & 13.0 & 59.8 & - & - & - & \\
\hline $\mathrm{COF}_{2}$ & - & - & - & 12.6 & 9.6 & 7.3 & 7.5 & 5.9 & 7.2 & 10.4 & 16.5 & 41.7 & 28.4 & 83.1 & - & - & - \\
\hline CFC-11 & 9.1 & 3.1 & 3.9 & 4.5 & 4.2 & 4.3 & 8.0 & 14.5 & - & - & - & - & - & - & - & - & - \\
\hline CFC-12 & 4.5 & 4.6 & 4.2 & 3.4 & 4.1 & 3.9 & 3.8 & 6.3 & 5.4 & 10.9 & 29.5 & 26.8 & - & - & - & - & - \\
\hline \multicolumn{18}{|c|}{ OR analysis } \\
\hline Alt $[\mathrm{km}]$ & 6.0 & 9.0 & 12.0 & 15.0 & 18.0 & 21.0 & 24.0 & 27.0 & 30.0 & 33.0 & 36.0 & 39.0 & 42.0 & 47.0 & 52.0 & 60.0 & 68.0 \\
\hline pres. & 7.0 & 6.5 & 8.4 & 7.4 & 4.9 & 4.2 & 4.4 & $4 . J$ & 3.2 & 2.8 & 4.2 & 3.3 & 4.6 & 4.0 & 12.0 & & 11.1 \\
\hline temp. & 2.04 & 1.46 & 1.21 & 0.96 & 1.07 & 0.98 & 0.85 & 1.15 & 0.74 & 0.91 & 1.15 & 1.27 & 1.66 & 2.41 & 4.14 & 4.10 & 5.52 \\
\hline $\mathrm{H}_{2} \mathrm{O}$ & 12.1 & 11.5 & 43.8 & 27.4 & 13.2 & 9.5 & 11.6 & 13.8 & 13.6 & 12.5 & 15.4 & 10.4 & 18.1 & 16.9 & 33.8 & 46.9 & - \\
\hline $\mathrm{O}_{3}$ & 23.8 & 38.8 & 20.0 & 15.3 & 7.6 & 5.3 & 6.1 & 6.2 & 5.0 & 7.0 & 7.8 & 5.6 & 10.2 & 9.9 & 14.5 & 26.8 & 25.4 \\
\hline $\mathrm{HNO}_{3}$ & 24.9 & 38.7 & 21.8 & 13.2 & 7.5 & 7.3 & 7.6 & 8.5 & 9.5 & 12.7 & 19.8 & 29.4 & 39.2 & 51.2 & 33.8 & - & - \\
\hline $\mathrm{CH}_{4}$ & 253 & 16.6 & 15.4 & 20.1 & 17.5 & 14.6 & 19.3 & 15.7 & 13.7 & 13.2 & 13.9 & 13.0 & 11.7 & 9.1 & 11.3 & 22.1 & 51.1 \\
\hline $\mathrm{N}_{2} \mathrm{O}$ & 50.0 & 12.4 & 10.4 & 11.4 & 10.9 & 10.6 & 11.7 & 11.2 & 10.6 & 12.0 & 11.3 & 10.7 & 14.4 & 14.1 & 47.0 & - & - \\
\hline $\mathrm{NO}_{2}$ & - & - & - & 34.1 & 29.8 & 11.3 & 9.8 & 10.8 & 11.0 & 8.9 & 8.8 & 7.9 & 9.0 & 16.9 & 97.5 & 53.1 & 36.2 \\
\hline
\end{tabular}

equilibrium, shape of the profiles outside the retrieval range, spectroscopic errors)

- instrumental errors (i.e. instrument line shape, radiometric and frequency calibration)

- influence of the interfering species onto the retrieved targets.

Details on how these errors have been evaluated can be found in Dudhia et al. (2002) and in the web site http://www. atm.ox.ac.uk/group/mipas/err/.

Table 3 reports, as a function of the retrieval altitudes, the total systematic errors affecting the retrieved values of all the targets included in the database for the FR and OR missions. As expected the systematic errors are different for the products derived from the FR and OR measurements, due to the different spectral region used in the retrievals and to the different measurement scenarios. We see that in general the systematic errors are higher for the OR data probably due to the fact that the reduced spectral resolution introduces correlations among the spectral features that cannot be removed even with a careful selection of the analysed spectral points. The total systematic errors for both FR and OR MWs are also reported in the database in specific files, one for each target. These files also report the major systematic error components contributing to the evaluated total systematic error.

\subsubsection{Individual information gain}

In order to asses the contribution of MIPAS observations to the atmospheric fields reported in MIPAS2D we have adopted a quantifier (called individual information gain) for the retrieved values of each target quantity (see Dinelli et al., 2009). The individual information gain is defined as:

$q_{j}=-\frac{1}{2} \log _{2}\left(\frac{S_{r j}}{S_{a j}}\right)$

where $S_{r j}$ is the diagonal element of the variance-covariance matrix (VCM) of the retrieval and $S_{a j}$ is the diagonal element of the VCM of the a-priori information for a particular profile point $j$ in the horizontal/vertical grid. $q_{j}$ provides a measure, in terms of bits of information, of the information gain provided by the analysed measurements with respect to the a-priori knowledge of each retrieved value (Rodgers, 2000). The value of $q_{j}$ depends on the size of the a-priori errors used in the retrieval, and therefore it is not an absolute quantifier. In regions where the information in the measurements is low, due to either cloud coverage or lack of atmospheric sampling, the retrieval error comes close to the a-priori error and the individual information gain value approaches zero. Any other value greater than zero indicates that some information about the retrieved quantity was extracted from the measurements. Our baseline is to consider $q_{j}$ values 
below 0.5 (that indicates that the a-priori error has been reduced by a factor of $\sqrt{2}$ ) as an indication that the retrieved value comes mainly from the a-priori knowledge of the target quantity.

Although retrieved values with low information gain are included in the database, these represent only a minor fraction as shown in Fig. 5 that reports the frequency at which a certain value of the individual information gain is obtained in the database. The figure shows that the individual information gain distributions peak at different values for the FR and OR data. This is due to the fact that the radiometric noise is different for the two missions (see Sect. 2.1). Since the noise level decreases from the FR to the OR measurements, the random errors (i.e. the squared root of the diagonal elements of the VCM of the retrievals) affecting the retrieved targets decrease on average of the same factor, while the error used for the a-priori is the same in the two analyses. The data with low values of $q_{j}$ can be filtered out a-posteriori by the users or properly handled through weighted averages (since they always correspond to poorly determined parameters) (see Sect. 4.5). It is however useful to keep in the database also values with low information gain because they may still be used in specific studies. As discussed below, negative values are artificially assigned to the individual information gain in order to flag data characterized by specific caveats.

Because of the large size of the files, the Averaging Kernel (AK) matrices relative to each analysed orbit are not included in the database. Only examples of the 2-D AK for both NOM and GRD grids are included in the database for both FR and OR missions; AKs for selected parts of the database can be supplied upon request.

\subsubsection{Single orbits and orbit segments}

We have seen in Sect. 4 that the best performance of the 2-D approach of GMTR is achieved when it is operated on a North Pole-to-North Pole complete orbit. Nevertheless, non-complete orbits, having a significant number of scans, are analysed and the results are included in the database because of their not-negligible fraction over the total number of MIPAS observations. Only orbit segments that cover a time period shorter than 20 min of continuous observations have been excluded from the analysis. In the case of NOM data, the horizontal grid used to retrieve the orbit segments is restricted to the geolocation range spanned by the available MIPAS scans. In the GRD case, the same horizontal grid, covering the complete orbit, has been used also for orbit segments; in this case, retrieved values laying outside the MIPAS measurement range are flagged with a negative value of the individual information gain (see also Sect. 4.5).

The quality of the results obtained with incomplete orbits was tested by analysing orbit segments artificially created from a full orbit. The comparison of the results of these analyses with those obtained from the corresponding

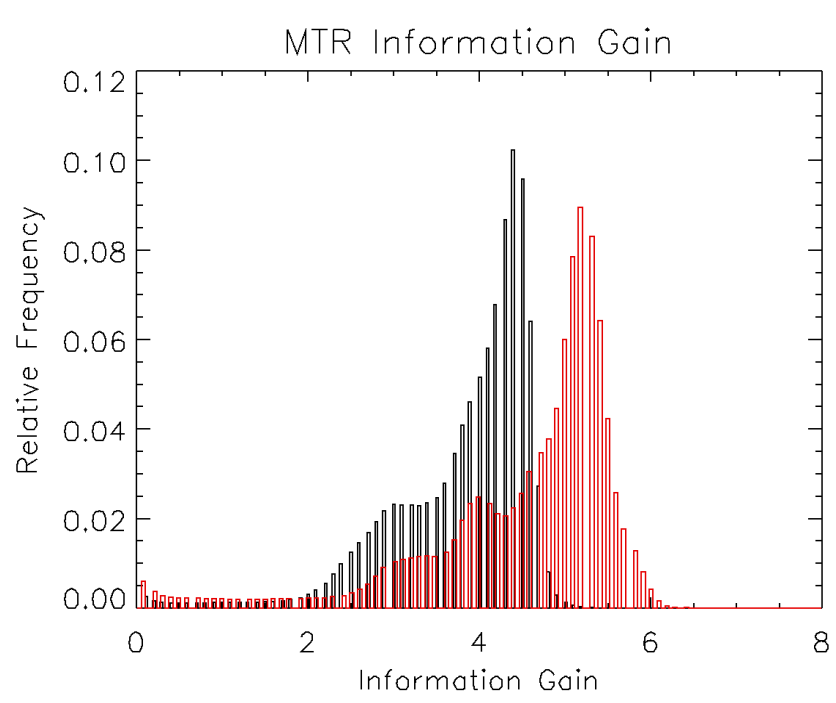

Fig. 5. Distribution of individual information gain values for temperature in the FR (black) and OR (red) GRD retrievals.

full orbit showed no significant deviations, apart from the atmospheric fields retrieved at the edges of the orbit segment. In MIPAS2D the data corresponding to these regions are properly characterized by low values of the corresponding individual information gain (see also the discussion on filtering in Sect. 4.5).

\subsubsection{Sensitivity to pointing calibrations}

Since GMTR does not retrieve pointing information, the impact on MIPAS2D results of the calibration routinely performed on the MIPAS instrument elevation angle needs to be investigated. During most of the FR mission, MIPAS pointing errors were affected by a significant seasonal cycle induced by a mispointing of the ENVISAT satellite. The pointing problem was first detected by von Clarmann et al. (2003) and attributed to a roll angle problem by Kiefer et al. (2007). This problem was corrected on December 2003 (orbit 9321) significantly improving the pointing stability. As a result, the vertical grid used as input to ESA Level 2 analyses is affected by seasonal and abrupt changes coinciding with pointing calibrations. The erroneous tangent altitude values reported in the Level $1 \mathrm{~b}$ files and their abrupt changes can also affect the results of the GMTR analysis, although the 2-D approach reduces the impact of the pointing error thanks to the compensation effect operated by the 2-D ray tracing of the multiple observation geometries that contribute to determine each profile. Nevertheless, some residual inconsistency remains in terms of pressure step-like changes in time series as shown in Fig. 6 that reports the evolution of FR daily mean pressure within the $20^{\circ} \mathrm{S}-20^{\circ} \mathrm{N}$ equatorial latitude band. Figure 6 also highlights the impact of the correction adopted in December 2003 when a more 


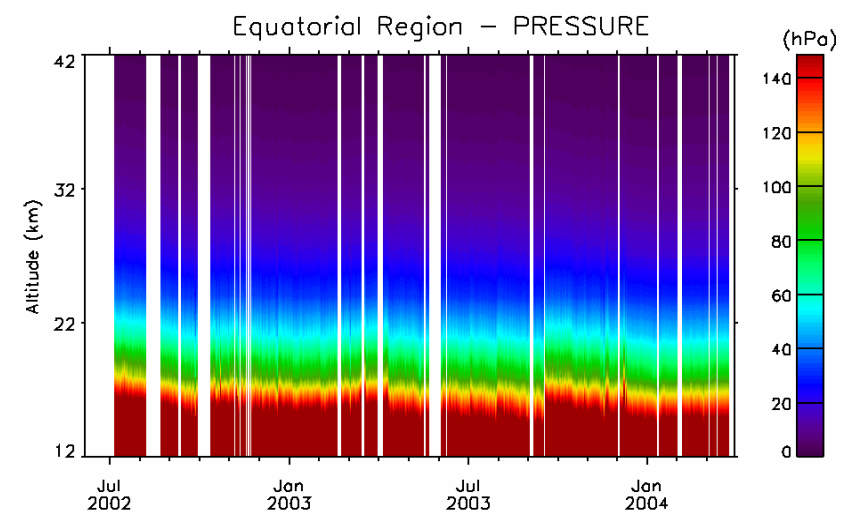

Fig. 6. Time series of FR daily mean pressures within the equatorial latitude band $\left(20^{\circ} \mathrm{S}-20^{\circ} \mathrm{N}\right)$.

stable pointing was achieved significantly improving the accuracy of the tangent altitudes hence reducing the pressure oscillations.

If MIPAS2D data are plotted versus altitude they appear to be partially affected by the pointing uncertainties.

\subsubsection{Comparison of NOM and GRD results}

The consistency between MIPAS2D products obtained in the two configurations of the horizontal grid (NOM and GRD) was tested using daily averages within the latitude bands selected for the development of the IG2 database by Remedios et al. (2007). For the majority of the data present in the two databases we have obtained a satisfactory agreement in terms of both average values and standard deviations. The averages were only marginally different in the most unfavourable cases of largest atmospheric variability (e.g. at the breaking of the southern polar vortex or in the disturbed winter northern polar region). However, even in these cases, the differences are mostly below the statistical error of the weighted average (and largely below the standard deviation), thus pointing towards a robust consistency. As an example of these comparisons, Fig. 7 shows mean profiles (obtained averaging the retrieved values weighted by their retrieval error) under typical conditions (temperatures in November - left panel) and under two worse-case scenarios (ozone at the breaking of the southern polar vortex - central panel, and $\mathrm{NO}_{2}$ in the disturbed winter North Pole - right panel). In each case, the mean profile (bold line) and the standard deviation (dashed line) are shown (black for NOM and red for GRD). Also shown are horizontal bars representing the weighted ESDs affecting each retrieved level of the profiles, although most are not visible because of their small size.

\subsection{Spatial resolution}

The FR and OR measurement strategies imply that the sampling of the atmosphere is not homogeneous throughout the whole MIPAS mission. The choice of a common retrieval grid (the GRD grid) for both missions was driven by the intent of producing a database where the spatial resolution of the retrieved values along the orbit track was as homogeneous as possible. Therefore the common retrieval grid was chosen to slightly undersample the atmosphere, in order to have a spatial resolution not driven by the geographical sampling of the instrument. Using the AK matrices it is possible to check whether this homogeneity has been achieved. MIPAS2D spatial resolution has been estimated performing retrievals on simulated observations. The observations were simulated using the LOS of real measurements, for both the FR and OR missions choosing one orbit per season, and assuming no cloud contamination. The atmospheric status used in the simulations was taken from the 2002 data of the IG2 database (Remedios et al., 2007) for the appropriate season. The spatial resolution of the retrieved values was estimated using the 2-D AK computed by GMTR at the end of the retrieval procedure, adopting the strategy described in Carlotti et al. (2007). Horizontal and vertical resolutions do not change from FR to OR data. The horizontal resolution is of the same size along the orbit for all targets (about $5.6^{\circ}$ ), while the vertical resolution has an altitude dependent value, depending on the distance between the altitudes chosen for the vertical retrieval grid. As an example, Fig. 8 shows a comparison of the average vertical and horizontal resolutions for the GRD retrieval grid for ozone in both FR and OR measurements for the four considered seasons. As shown in the figure, the horizontal resolution does not change from FR to OR missions. The vertical resolution shows the same behaviour with altitude for the two missions, apart for the lowermost altitudes, where the OR data show a much worse performance, due to the fact that in a large part of the orbit there are no measurements going down to $6 \mathrm{~km}$.

It should be noted that, in order to compensate for the orbit inclination (of $8.9^{\circ}$ ) the MIPAS LOSs deviate from the orbit plane. This deviation, that varies along the orbit, constraints the Geo-fit to assume atmospheric homogeneity across the orbit track. The latitudinal component of this deviation extends up to a maximum of about $2^{\circ}$ at tangent points. This implies that the Geo-fit analysis cannot be forced to horizontal resolutions better than $2^{\circ}$ in latitude. The horizontal resolution of our products, as it can be seen in Fig. 8 is about 5.6 latitudinal degree, a value wider than the maximum latitudinal deviation due to the inclination of the orbit. As for the longitudinal component, it is commonly accepted that atmospheric variability is minor when moving at adjacent longitudes. However, in case of extreme events, such as non-centered polar vortex or Asian monsoon anticyclone, the variability may assume not-negligible values, introducing systematic errors difficult to assess. 

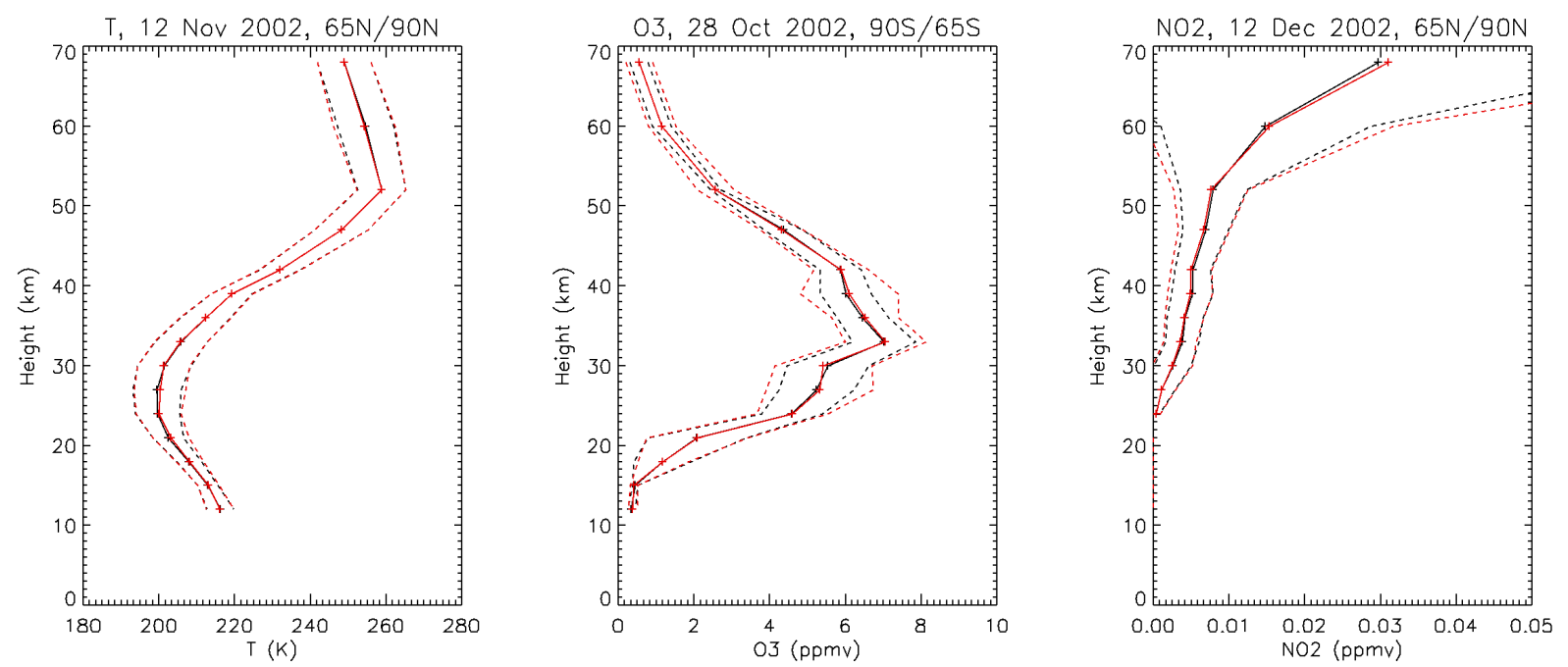

Fig. 7. Consistency between the results of the NOM and GRD datasets adopting different horizontal grid. Black lines represent the NOM data and red lines the GRD data. Solid lines represent the averaged values and dashed lines represent their standard deviation. Weighted ESDs are shown with horizontal lines at each altitude. Left panel: temperature at the North Pole in November 2002. Centre: ozone at the South Pole in late October 2002. Right: $\mathrm{NO}_{2}$ at the North Pole in December 2002.

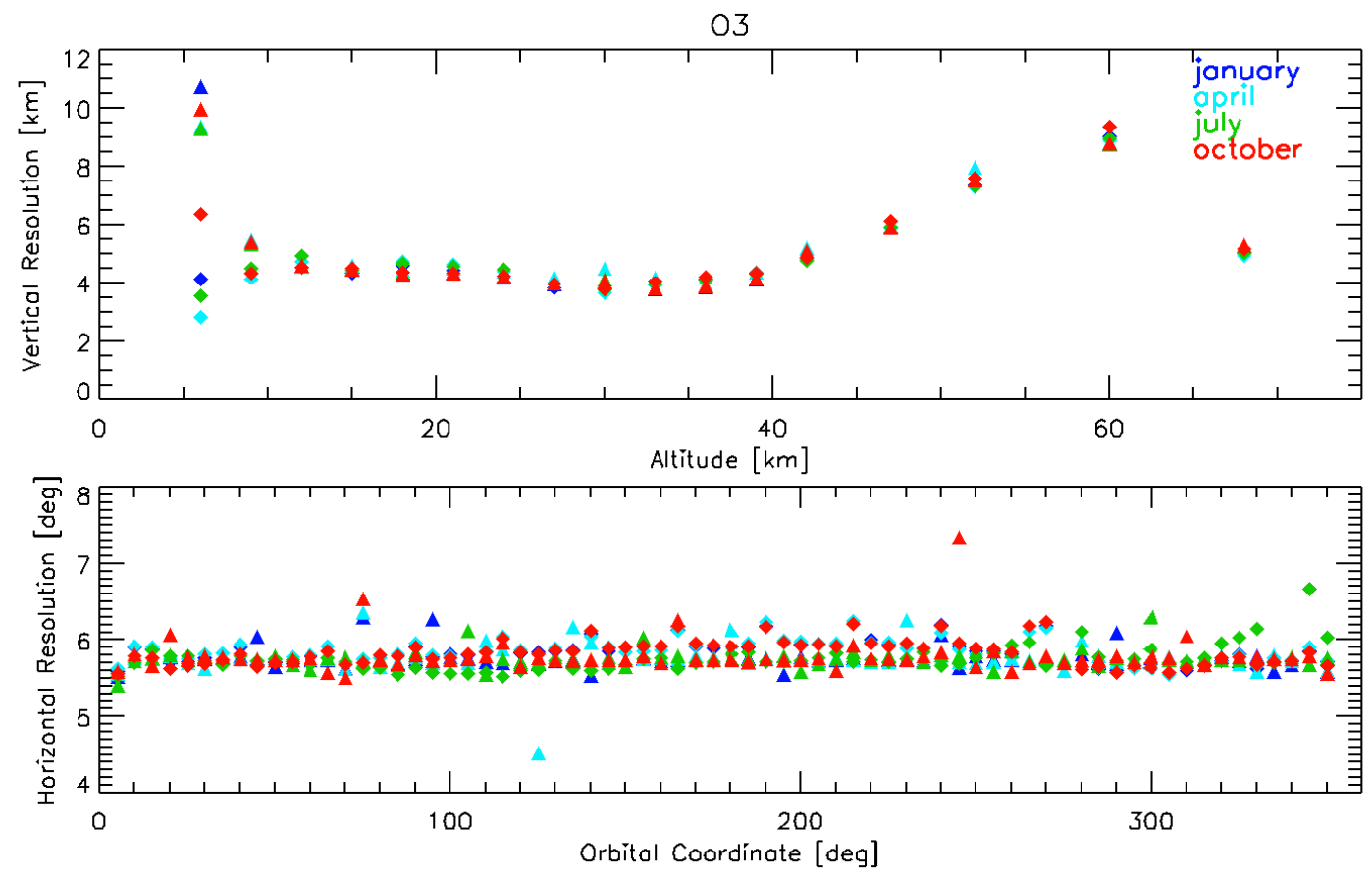

Fig. 8. Comparison of ozone vertical (top panel) and horizontal (bottom panel) averaged resolutions computed for the FR (diamonds) and OR (triagles) missions. The colours identify the season as reported in the legend. 


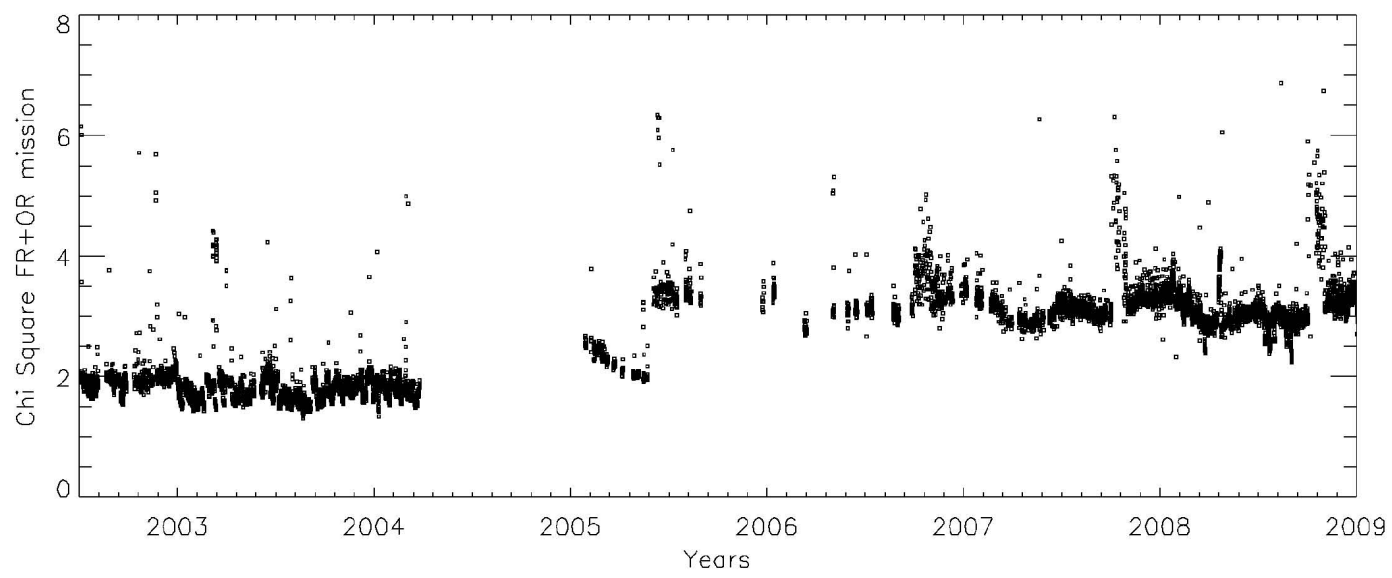

Fig. 9. $\chi^{2}$ values of the MTR retrievals for the GRD dataset plotted as a function of acquisition time.

\subsection{Filtering and consolidated datasets}

The whole MIPAS mission was analysed with GMTR using an automated procedure. Before including the final results into the database, the quality of the results was checked, in order to remove corrupted and low quality data. At the time of writing, MIPAS measurements up to December 2008 have been analysed and only the filtered results have been included into the database. In total 7188 FR orbits and 8057 OR orbits have been analysed. As discussed in Sect. 2 part of the FR and of the OR missions were measured adopting special observation modes that are not analysed in this first release of MIPAS2D. Furthermore a fraction of the nominal mode measurements was discarded because of either failures in reaching convergence or poor quality of the retrievals often due to poor Level $1 \mathrm{~b}$ data. As a result of the data quality filtering, the FR_NOM dataset includes 5830 orbits, while the GRD dataset includes 5830 FR orbits and 6843 OR orbits.

Considering that MIPAS2D can be used for a variety of applications, the filtering stage was tuned at a level that enables to exclude products that appear to be unphysical but not those that provide unexpected values of the retrieved quantities. The filtering criteria are based on the quality of the spectral fitting, on threshold values of the information gain (see Sect. 4.3.2) and on the presence of Level 1b corrupted data. The first stage of filtering was on the value of the final $\chi^{2}$, defined as the normalized $L_{2}$ noise weighted norm of the residuals (observed minus simulated spectral radiances) at the final step of the iterative retrieval procedure (see Eq. 9 of Ridolfi et al., 2007). This quantifier provides a measure of how well the results of the forward model simulations are able to reproduce the measurements of the full orbit taking into account the measurement errors. A $\chi^{2}$ equal to 1 indicates that the spectral residuals are consistent with the measurement noise level. The filtering was operated adopting a threshold of 7 on the $\chi^{2}$ of the MTR retrieval of pressure, temperature, water vapour and ozone. No filtering was applied to the other targets based on the $\chi^{2}$ value. Although a high $\chi^{2}$ does not imply that the retrieved quantities are not realistically describing the analysed spectrum, high values were found to be associated with orbits having whole parts affected by poor Level $1 \mathrm{~b}$ data. Since all the orbits leading to a MTR $\chi^{2}$ above 7 were discarded, all other targets are consequently not available for these orbits. On the other hand, targets with a day-night variability such as $\mathrm{NO}_{2}$ can lead to high $\chi^{2}$ values coincident to low $\chi^{2}$ values of the MTR analysis of the same orbit: these products were all kept in the database. Most of the rejected orbits have failed to reach an acceptable convergence during the retrieval. The final $\chi^{2}$ for the MTR retrieval of pressure, temperature, water vapor and ozone for the full MIPAS mission (GRD dataset) is shown in Fig. 9. The figure shows the long term self-consistency of the FR and OR datasets, with no indication of a trend or increased spread along the extension of the MIPAS mission. Figure 9 also highlights the effects of the different noise levels of the FR and OR missions; actually the $\chi^{2}$ value is negatively correlated with the absolute value of the noise (see Fig. 2), indicating that the retrievals of both FR and OR measurements are affected by systematic errors, that cannot be compensated for by the retrieval procedure, of similar size.

Apart from the automatic removal in the retrieval iterations of negative values, the MIPAS2D results are intentionally not filtered against anomalous values or strong deviations from climatological expectations in order to avoid the introduction of biases and the loss of information if anomalous atmospheric events are encountered. This choice however implies that the user should take some caution in using the database. Unless seeking specific data under very unfavourable situations, information gain below 0.5 (see Sect. 4.3.2) is a proxy for poor retrieval information, and the user should carefully evaluate the impact of the use of these data. As an example, the distribution of information gain for temperature results shown in Fig. 5 shows that the 

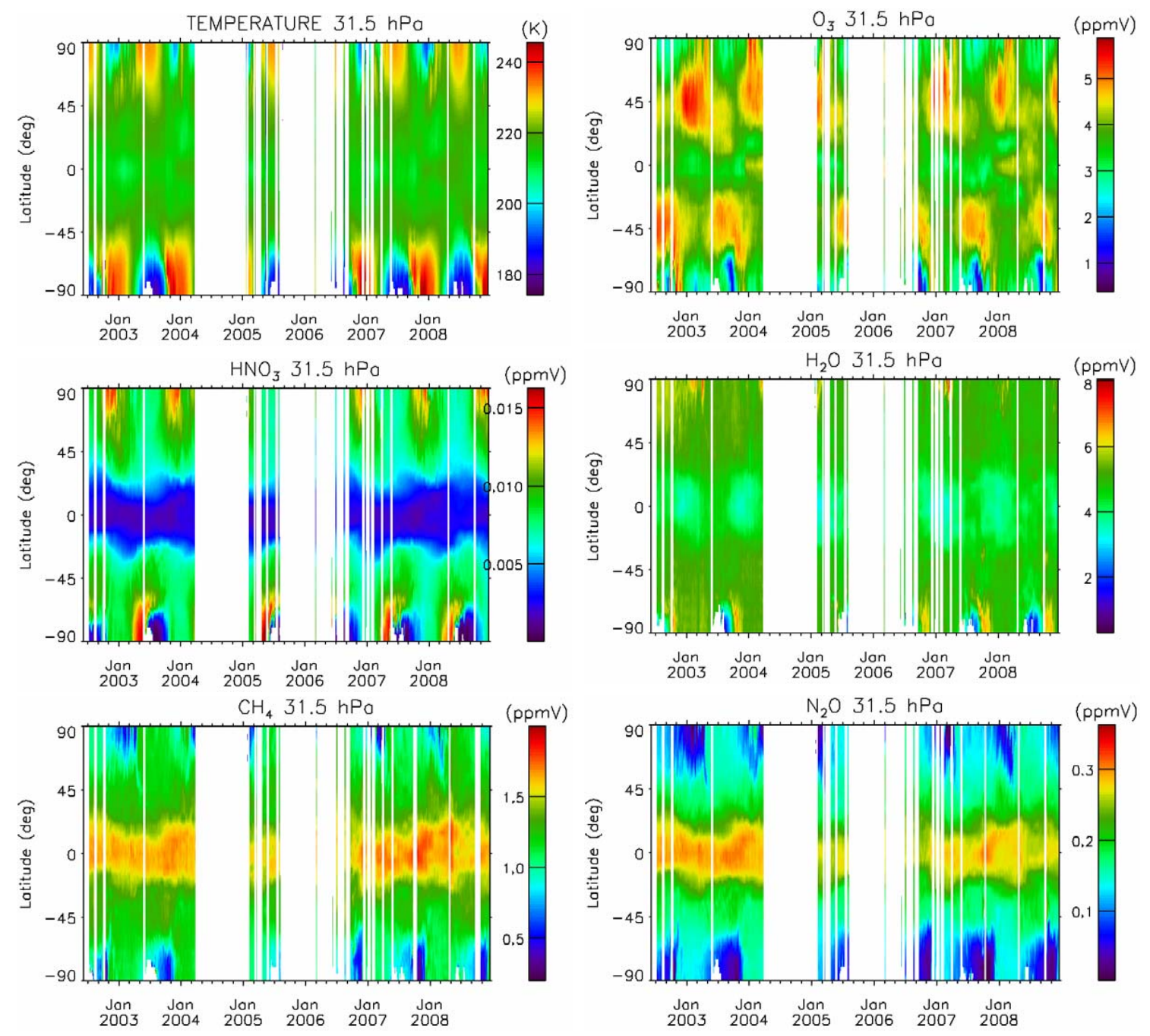

Fig. 10. 5-days means of temperature, ozone, $\mathrm{HNO}_{3}$, water, methane and $\mathrm{N}_{2} \mathrm{O}$, mapped as a function of time and Latitude for the pressure level of $31.5 \mathrm{hPa}(24 \mathrm{~km})$.

suggested thresholds on the information gain affect only a minor fraction of the datasets.

Further filtering of the data was performed on the basis of the consistency of the values of the information gain. Because of numerical instabilities in the matrix inversion algorithm, the retrieval error for some parameters assumes unrealistically very small values, consequently their information gain assumes unacceptable high values. These data were removed flagging them with negative values of the information gain. In the case of the GRD dataset, negative information gain is also used as a quality flag to identify retrieved data that are located outside the range of MIPAS observations (see Sect. 4.3.3). We stress again that the results flagged with negative information gain should be discarded by the end users since they are kept in the database only for the sake of uniformity of the released products.
As a consequence of the chosen strategy to originate and close the orbits at the North Pole the profiles in the arctic region are derived from measurements that are not consecutive in time. The time lag between the data determined before and after the North Pole is about 100 minutes; for this reason the users should be careful in the usage of the TIME information reported in the files of the database. If required, for selected cases it is possible to repeat the analysis originating and closing the orbits at the South Pole.

\section{Examples of results}

As examples of the potentiality of MIPAS2D we show some possible applications of the dataset. Figure 10 shows time series relative to six targets obtained with both FR and OR MIPAS2D results. The maps in Fig. 10 report average values 


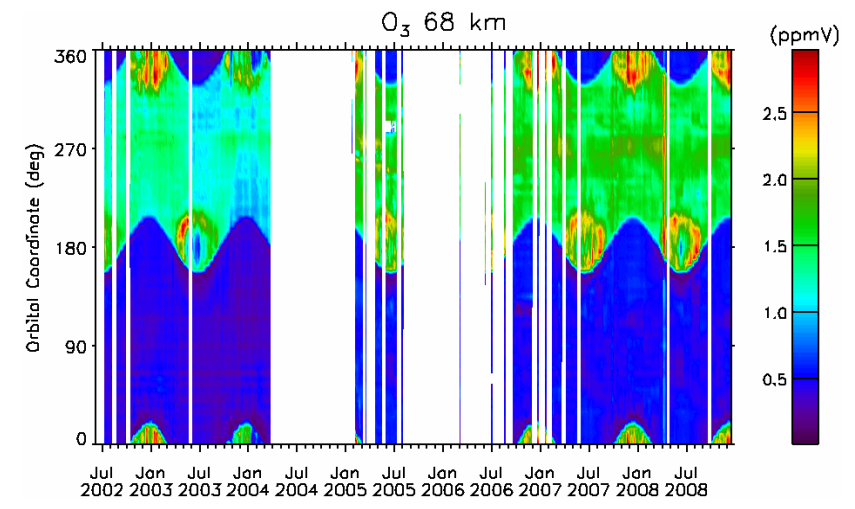

Fig. 11. 5-days mean of $\mathrm{O}_{3} \mathrm{VMR}$ (ppmv) at $68 \mathrm{~km}$ plotted as a function of the $\mathrm{OC}$ and the acquisition time.

computed over a 5 days period (this averaging criterion will be used also for Figs. 11 and 12) at the pressure level of $31.5 \mathrm{hPa}$ (about $24 \mathrm{~km}$ ) and for latitudinal bins of $5^{\circ}$. The averaged values for temperature, $\mathrm{H}_{2} \mathrm{O}, \mathrm{O}_{3}, \mathrm{HNO}_{3}$, $\mathrm{CH}_{4}$ and $\mathrm{N}_{2} \mathrm{O}$ VMRs have been weighted with their noise error and only data with $q \geq 0.5$ have been included in the average. White stripes refer to time periods where the data availability was too low to obtain averages. The plots shown in Fig. 10 highlight the long term time coverage of MIPAS observations, showing two main data gaps. The first gap extends from April 2004 to January 2005 when MIPAS was switched off. The second data-gap starts in August 2005 and extends to September 2006; this is mainly due to a low duty cycle (around 35\%) and a limited number of observations performed with the nominal observation mode.

MIPAS main targets included in the database can be used to study the chemistry and dynamics of the region extending from the upper troposphere to the lower mesosphere. The temperature map of Fig. 10 shows the evolution of the polar vortices over the years, with colder temperatures for the Southern Polar winter than for the Northern Polar winter. The ozone map shows a clear depletion at the South Pole in September, ending at the breaking up of the vortex. The $\mathrm{HNO}_{3}$ map shows a build up at the South Pole in April, May and June, then a depletion corresponding to the winter period with a progressive increase up to the breaking of the polar vortex. We also see the seasonal alternation of high and low peak ozone values at mid latitudes. The water vapor map shows the dehydration occurring at the South Pole during spring and at the tropics around January each year. $\mathrm{CH}_{4}$ and $\mathrm{N}_{2} \mathrm{O}$, which can be used as trace species for the dynamics of the stratosphere, show similar latitudinal behavior. Both gases show a seasonal latitudinal displacement of their peak values within the region edged by the subtropical dynamical barriers, as the meridional circulation changes with season. This behaviour is seen also in $\mathrm{HNO}_{3}$ but with an opposite sign of the horizontal gradient.

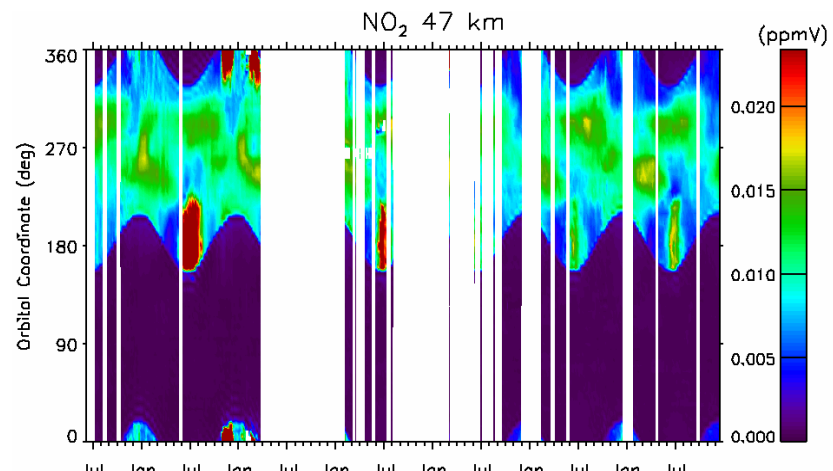

Jul Jon Jul Jon Jul Jon Jul Jon Jul Jon Jul Jon Jul

Fig. 12. 5-days means of $\mathrm{NO}_{2} \mathrm{VMR}$ (ppmv) at $47 \mathrm{~km}$ plotted as a function of the $\mathrm{OC}$ and the acquisition time.

In the GMTR analyses most of the initial part of the reconstructed orbits, and therefore OC below $180^{\circ}$, lies in the dayside. The exact range of the $\mathrm{OC}$ lying in the dayside depends on the solar zenith angle and therefore varies with season. Figure 11 shows the time series of the ozone VMR values at $68 \mathrm{~km}$ on the orbital coordinates of the GRD retrieval grid. At this altitude the photolysis of ozone into atomic oxygen leads to order of magnitude differences between daytime and nightime concentrations of ozone. This leads to the abrupt changes at the day-night terminators and the depicted seasonal evolution (Brasseur and Solomon, 2005).

Figure 12 shows the $\mathrm{NO}_{2}$ VMR time series at $47 \mathrm{~km}$ on the orbital coordinates of the GRD retrieval grid. $\mathrm{NO}_{2}$ concentrations are very low during the day time while they suddenly increase at dusk, with larger changes at the South Pole than at the North Pole. Events of abrupt $\mathrm{NO}_{2}$ enhanced concentrations are visible at the winter poles (e.g. July 2003 at the South Pole) due to downward transport of mesospheric $\mathrm{NO}_{2}$ (see Funke et al., 2005) or during solar proton events (e.g. at both poles in October-November 2003, see López-Puertas et al., 2005).

Figures 13-15, show time series of 10-days averages of temperature (top panels) and ozone VMR (bottom panels) from 200 to $0.2 \mathrm{hPa}$ (about 12 to $60 \mathrm{~km}$ in altitude), respectively. In Fig. 13 the maps refer to the antarctic $\left(65^{\circ} \mathrm{S}-90^{\circ} \mathrm{S}\right)$ region, in Fig. 14 to the equatorial $\left(20^{\circ} \mathrm{S}-20^{\circ} \mathrm{N}\right)$ region and in Fig. 15 to the arctic $\left(65^{\circ} \mathrm{N}-90^{\circ} \mathrm{N}\right)$ region. These examples show the dynamical and chemical evolution discussed above throughout the whole vertical range covered by the observations.

Figure 16 shows the results of the GMTR analysis on the NOM grid (FR mission only) and reports the time series for the VMR of minor species $\mathrm{N}_{2} \mathrm{O}_{5}, \mathrm{ClONO}_{2}, \mathrm{COF}_{2}, \mathrm{CFC}-11$ and $\mathrm{CFC}-12$. All these maps show the 10-days average of the data in the antarctic latitudinal band $\left(65^{\circ} \mathrm{S}-90^{\circ} \mathrm{S}\right)$. For these species the vertical coverage of the analysis has to be reduced due to the very low information content of the measurements 


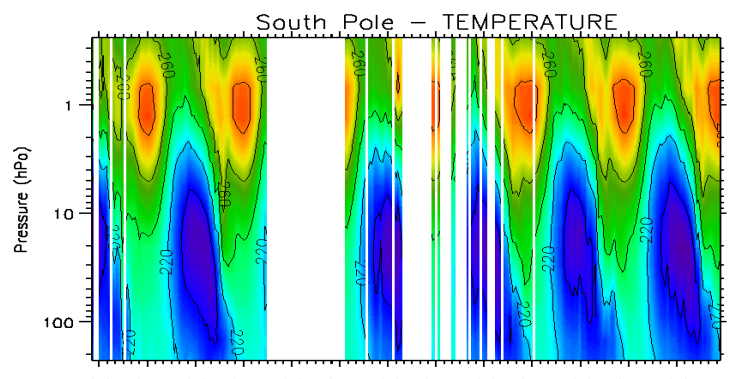

Jul Jan Jul Jon Jul Jon Jul Jon Jul Jan Jul Jan Jul
2002200320032004200420052005200620062007200720082008

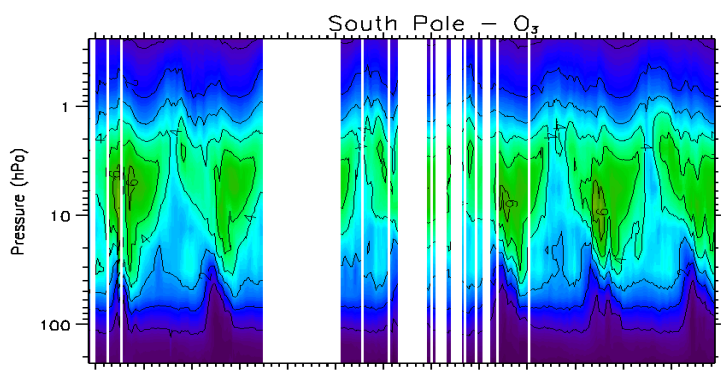

Jul Jan Jul Jon Jul Jon Jul Jon Jul Jon Jul Jon Jul
2002200320032004200420052005200620062007200720082008

Fig. 13. Time series of 10-days means of temperature and ozone in the Antarctic region $\left(65^{\circ} \mathrm{S}-90^{\circ} \mathrm{S}\right)$.

outside the plotted range. The figure highlights the seasonal evolution of the species in the Southern Polar region: The timeseries of CFCs and of the relatively stable $\mathrm{COF}_{2}$ show a strong reduction during polar night in coincidence with the descent of mesospheric air in the polar vortex. In the descending mesospheric air, nitrogen oxides reservoir species $\mathrm{N}_{2} \mathrm{O}_{5}$ and $\mathrm{ClONO}_{2}$ show large increases which couple with those of $\mathrm{NO}_{2}$ as seen in Fig. 12. The combined study of these molecules aids in tracing the different effects of transport and chemistry on this region, and in particular on ozone chemistry.

A further example of the potentiality of the MIPAS2D database in terms of applicability to stratospheric dynamics is shown in Fig. 17 where the ozone VMR at $21 \mathrm{~km}$ is reported for the measurements acquired from 13 to 16 September 2002 (left hand panel) and from 23 to 26 of the same month (right hand panel) when the Southern polar vortex splitted into two lobes for the first time ever recorded (Simmons et al., 2003). The split of the polar vortex around the 24 September is clearly traced by the split of the ozone hole.

MIPAS2D has been exploited to study the influence of transient luminous events on the atmosphere (Arnone et al., 2009b) and to analyze trends of ozone and several molecular species related to ozone chemistry: $\mathrm{HNO}_{3}, \mathrm{~N}_{2} \mathrm{O}, \mathrm{NO}_{2}$, $\mathrm{N}_{2} \mathrm{O}_{5}, \mathrm{ClONO}_{2}, \mathrm{COF}_{2}, \mathrm{CFC}-11$ and CFC-12 by Papandrea et al. (2010). Starting from January 2008 MIPAS duty cycle was increased back up to $100 \%$ and the mission has been extended to the end of 2013. Therefore the
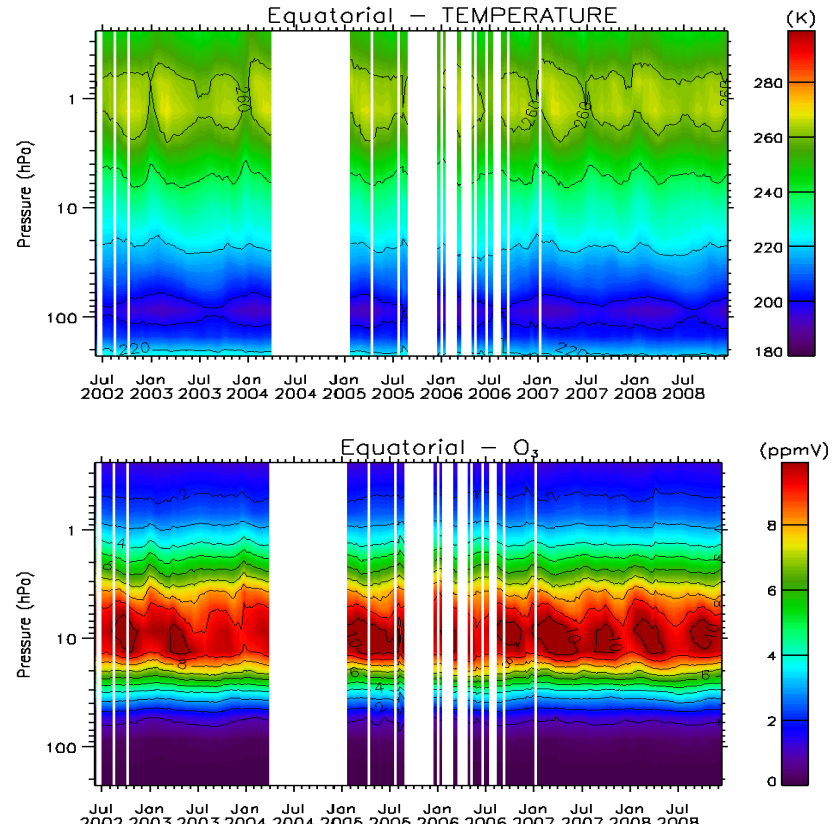

Fig. 14. Time series of 10-days means of temperature and ozone in the Equatorial region $\left(20^{\circ} \mathrm{S}-20^{\circ} \mathrm{N}\right)$.
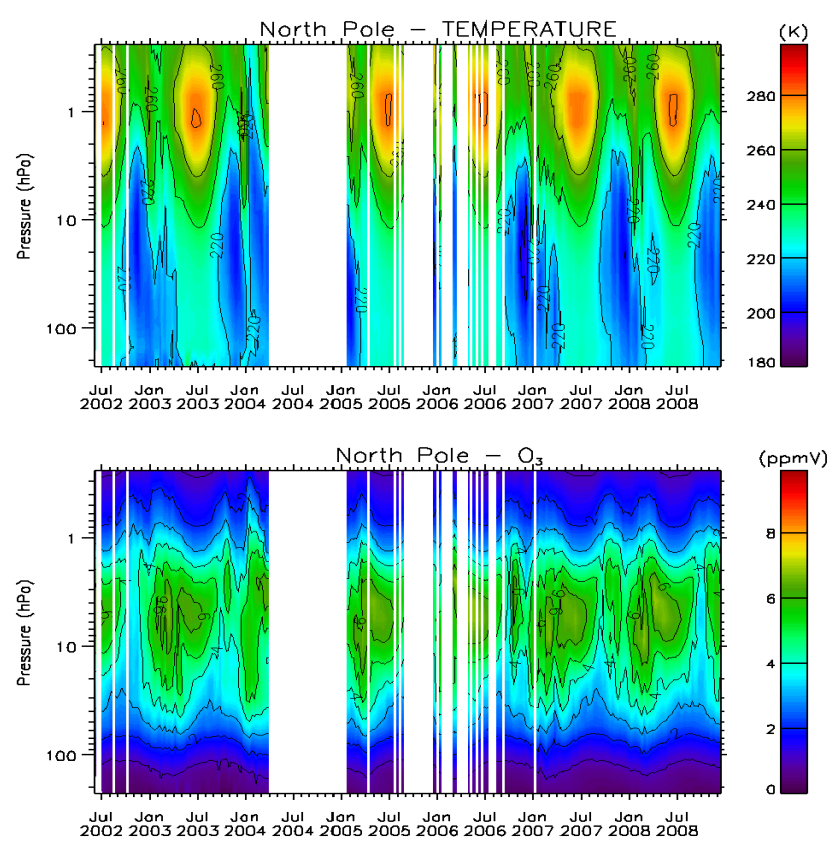

Fig. 15. Time series of 10-days means of temperature and ozone in the Arctic region $\left(65^{\circ} \mathrm{N}-90^{\circ} \mathrm{N}\right)$. 

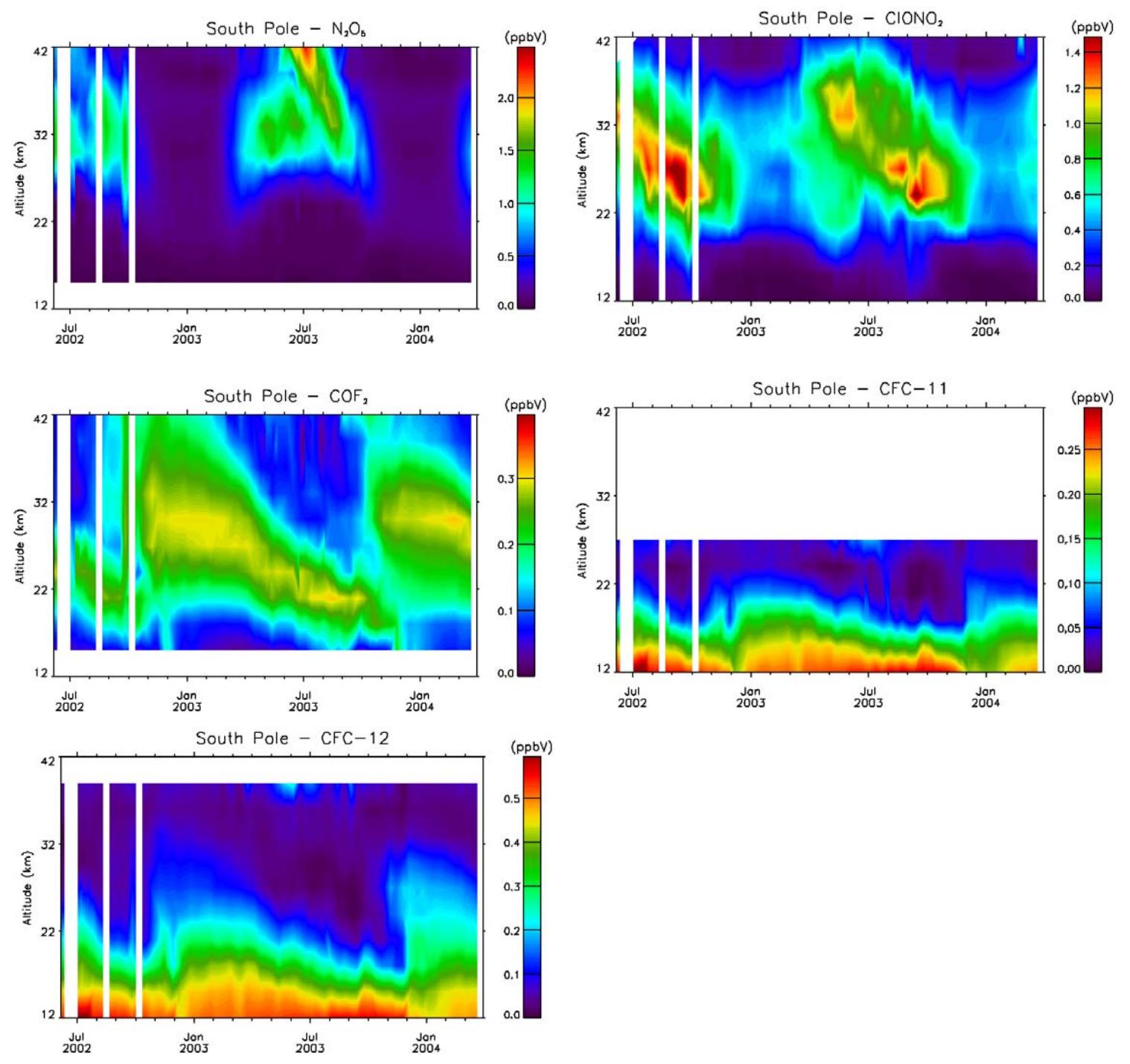

Fig. 16. Time series of 10-days means for the minor species $\mathrm{N}_{2} \mathrm{O}_{5}, \mathrm{ClONO}_{2}, \mathrm{COF}_{2}, \mathrm{CFC}-11$ and $\mathrm{CFC}-12$ in the Antarctic region $\left(65^{\circ} \mathrm{S}-\right.$ $90^{\circ} \mathrm{S}$ ) mapped as a function of altitude and acquisition time.

analysis strategy adopted in that paper is well suited also for a refined analysis and a correct monitoring of possible ozone recovery, as required by the Montreal protocol and successive amendments as well as shorter term studies.

\section{Conclusions and future development of the database}

We have analysed all the measurements acquired by MIPAS in its nominal observation mode from the beginning of the mission up to December 2008 by applying for the first time a tomographic approach to such an extended set of data. The obtained MIPAS2D database contains 2-D fields of pressure, temperature and VMR of $\mathrm{H}_{2} \mathrm{O}, \mathrm{O}_{3}, \mathrm{HNO}_{3}$, $\mathrm{CH}_{4}, \mathrm{~N}_{2} \mathrm{O}$ and $\mathrm{NO}_{2}$ that have been obtained on a fixed altitude-latitude grid for the whole mission. The analyses

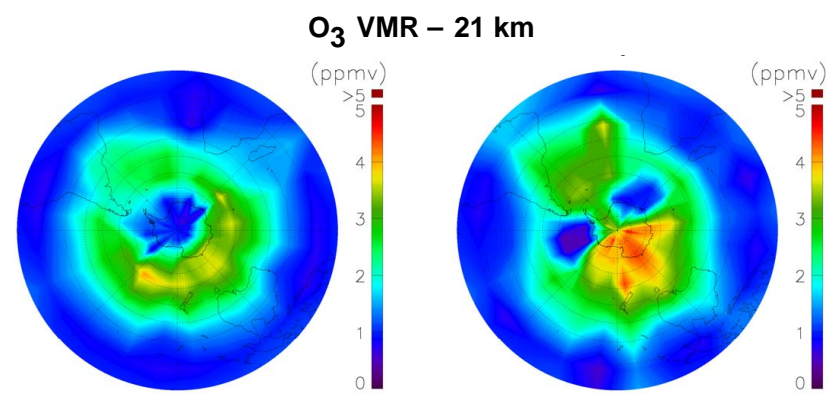

Fig. 17. Ozone VMR (ppmv) at $21 \mathrm{~km}$ altitude in the South Pole region. The left panel shows the data measured on 13-16 September 2002, while the right panel shows the same region measured on 23-26 September 2002. The vortex split is clearly visible. 
have been perfomed with the GMTR system that enables the tomographic retrieval of MIPAS data taking properly into account the horizontal inhomogeneities of the atmosphere.

The MIPAS2D database is intended to promote the use of MIPAS products and stimulate scientific collaboration. The full database (or part of it) can be downloaded from the web sites http://www.mbf.fci.unibo.it/mipas2d.html, or http://www.isac.cnr.it/ rss/mipas2d.htm. These web pages contain the technical instructions for the users and are updated in the case of new releases or when new products are available in the database. The files can be downloaded with login credentials that are obtained by sending an e-mail to mipas2d@fci.unibo.it.

For the FR mission, the database is extended with the 2-D distribution on the NOM grid of the targets mentioned above complemented with the VMRs of $\mathrm{N}_{2} \mathrm{O}_{5}, \mathrm{ClONO}_{2}$, $\mathrm{COF}_{2}$, CFC-11, and CFC-12. To date these minor species have been retrieved for the FR mission only. Other target species, whose emission spectrum falls into the frequency range measured by MIPAS, can be retrieved on demand. The obtained results are complemented with detailed auxiliary files. The database is continuously updated with the analysis of new available Level $1 \mathrm{~b}$ files of the OR mission, and is being expanded to other MIPAS special observation modes.

Acknowledgements. E. Arnone acknowledges funding through the European Community's Human Potential Programme Marie Curie under contract MERG-CT-2007-209157. E. Papandrea acknowledges support by ESA within the framework of the Changing Earth Science Network Initiative.

Edited by: T. von Clarmann

\section{References}

Arnone, E., Kiefer, M., Carlotti, M., Castelli, E., von Clarmann, T., Dinelli, B. M., Höpfner, M., Kellmann, S., Linden, A., Papandrea, E., Ridolfi, M., and Stiller, G.: Impact Of Horizontal Temperature Gradient On CFC-11 Fields Retrieved From MIPAS Limb Sounding: One-Dimensional Approach Versus Two-Dimensional Tomography, ASSFTS-14, Florence May 2009, 2009a.

Arnone, E., Kero, A., Enell, C.-F., et al.: Seeking sprite-induced signatures in remotely sensed middle atmosphere $\mathrm{NO}_{2}$ : latitude and time variations, Plasma Sources Sci. Technol., 18, 034014, doi:10.1088/0963-0252/18/3/034014, 2009b.

Brasseur, G. P. and Solomon, S.: Aeronomy of the Middle Atmosphere, 3rd edn., Springer, Dordrecht, The Netherlands, 2005.

Carlotti, M.: Global-fit approach to the analysis of limb-scanning atmospheric measurements, Appl. Opt., 27, 3250-3254, 1988.

Carlotti, M., Dinelli, B. M., Raspollini, P., and Ridolfi, M.: Geo-fit Approach to the Analysis of Limb-Scanning Satellite Measurements, Appl. Opt., 40, 1872-1885, 2001.
Carlotti, M., Brizzi, G., Papandrea, E., Prevedelli, M., Ridolfi, M., Dinelli, B. M., and Magnani, L.: GMTR: Two-dimensional multi-target retrieval model for MIPAS-ENVISAT observations, Appl. Opt., 45(4), 716-727, 2006.

Carlotti, M., Dinelli, B. M., Papandrea, E., Ridolfi, M.: Assessment of the horizontal resolution of retrieval products derived from MIPAS observations, Opt. Express, 15(16), 10458-10472, 2007.

Ceccherini, S., Mencaraglia, F., Geophysical validation tests, Technical note IFAC-GA-2004-18-SC of the ESA study "Development of Algorithm for the Exploitation of MIPAS Special Modes Measurements" ESA-ESRIN Contract No. 16700/02/I-LG, 2004.

Dinelli, B. M., Alpaslan, D., Carlotti, M., Magnani, L., and Ridolfi, M.: Multi-Target retrieval (MTR): the simultaneous retrieval of pressure, temperature and volume mixing ratio profles from limb-scanning atmospheric measurements, J. Quant. Spectrosc. Radiat. Transf., 84, 141-157, 2004.

Dinelli, B. M., Castelli, E., Carli, B., Del Bianco, S., Gai, M., Santurri, L., Moyna, B. P., Oldfield, M., Siddans, R., Gerber, D., Reburn, W. J., Kerridge, B. J., and Keim, C.: Technical Note: Measurement of the tropical UTLS composition in presence of clouds using millimetre-wave heterodyne spectroscopy, Atmos. Chem. Phys., 9, 1191-1207, 2009, http://www.atmos-chem-phys.net/9/1191/2009/.

Dudhia, A., Jay, V. L., and Rodgers, C. D.: Microwindow selection for high-spectral-resolution sounders, Appl. Opt., 41(18), 36653673, 2002.

Dudhia, A. and Piccolo, C.: MIPAS Observations of Stratospheric Trends, Proceedings of the ESA Envisat Symposium 2007, Montreux, Switzerland, 22-27 April 2007, 2007.

Dudhia, A.: MIPAS-related Section of the web-site of the Oxford University: www.atm.ox.ac.uk/group/mipas, 2008.

Fischer, H., Birk, M., Blom, C., Carli, B., Carlotti, M., von Clarmann, T., Delbouille, L., Dudhia, A., Ehhalt, D., Endemann, M., Flaud, J. M., Gessner, R., Kleinert, A., Koopman, R., Langen, J., López-Puertas, M., Mosner, P., Nett, H., Oelhaf, H., Perron, G., Remedios, J., Ridolfi, M., Stiller, G., and Zander, R.: MIPAS: an instrument for atmospheric and climate research, Atmos. Chem. Phys., 8, 2151-2188, 2008, http://www.atmos-chem-phys.net/8/2151/2008/.

Flaud, J.-M., Piccolo, C., Carli, B., Perrin, A., Coudert, L. H., Teffo, J.-L., and Brown, L.R.: Molecular line parameters for the MIPAS (Michelson Interferometer for Passive Atmospheric Sounding) experiment, Atmos. Oceanic Opt., 16, 172-182, 2003.

Funke, B., López-Puertas, M., Gil-López, S., von Clarmann, T., Stiller, G. P., Fischer, H., Kellmann, S., Downward transport of upper atmospheric $\mathrm{NO}_{\mathrm{x}}$ into the polar stratosphere and lower mesosphere during the Antarctic 2003 and Arctic 2002/2003 winters, J. Geophys. Res., 110(D24), D24308, doi:10.1029/2005JD006463, 2005.

Kiefer, M., von Clarmann, T., Grabowski, U., De Laurentis, M., Mantovani, R., Milz, M., and Ridolfi, M.: Characterization of MIPAS elevation pointing, Atmos. Chem. Phys., 7, 1615-1628, 2007, http://www.atmos-chem-phys.net/7/1615/2007/.

Kleinert, A., Aubertin, G., Perron, G., Birk, M., Wagner, G., Hase, F., Nett, H., and Poulin, R.: MIPAS Level 1B algorithms overview: operational processing and characterization, Atmos. Chem. Phys., 7, 1395-1406, 2007 , http://www.atmos-chem-phys.net/7/1395/2007/. 
Kühl, S., Puķīte, J., Deutschmann, T., Platt, U. and Wagner, T., SCIAMACHY limb measurements of $\mathrm{NO}_{2}, \mathrm{BrO}$ and OClO. Retrieval of vertical profiles: Algorithm, first results, sensitivity and comparison studies, Adv. Space Res., 42, 1747-1764, 2008.

Livesey, N. J., Van Snyder, W., Read, W. G., and Wagner, P. A.: Retrieval algorithms for the EOS Microwave Limb Sounder (MLS), IEEE T. Geosci. Remote, 44, 1144-1155, 2006.

López-Puertas, M., Funke, B., Gil-López, S., von Clarmann, T., Stiller, G. P., Höpfner, M., Kellmann, S., Fischer, H., and Jackman, H. C., Observation of $\mathrm{NO}_{x}$ Enhancement and Ozone Depletion in the Northern and Southern Hemispheres after the October-November 2003 Solar Proton Events, J. Geophys. Res., 110(A9), A09S43, doi:10.1029/2005JA011050, 2005.

Milz, M., von Clarmann, T., Fischer, H., Glatthor, N., Grabowski, U., Höpfner, M., Kellmann, S., Kiefer, M., Linden, A., Mengistu Tsidu, G., Steck, T., Stiller, G. P., Funke, B., and López-Puertas, M.: Water vapour distributions measured with the Michelson Interferometer for Passive Atmospheric Sounding on board Envisat (MIPAS/Envisat), J. Geophys. Res., 110(D24), D24307, doi:10.1029/2005JD005973, 2005.

Oelhaf H.: MIPAS Mission Plan, Issue 4, Version 3, ESA Technical Note ENVI-SPPA-EOPG-TN-07-0073, January 2008, 2008

Papandrea, E., Arnone, E., Brizzi, G., Carlotti, M., Castelli, E., Dinelli, B. M., and Ridolfi, M.: Two-dimensional tomographic retrieval of MIPAS/ENVISAT measurements of ozone and related species, Int. J. Rem. Sens., 31, 477-483, doi:10.1080/01431160902893501, 2010.

Puķīe, J., Kühl, S., Deutschmann, T., Platt, U., and Wagner, T.: Accounting for the effect of horizontal gradients in limb measurements of scattered sunlight, Atmos. Chem. Phys., 8, 3045-3060, 2008, http://www.atmos-chem-phys.net/8/3045/2008/.

Raspollini, P., Belotti, C., Burgess, A., Carli, B., Carlotti, M., Ceccherini, S., Dinelli, B. M., Dudhia, A., Flaud, J.-M., Funke, B., Hpfner, M., López-Puertas, M., Payne, V., Piccolo, C., Remedios, J. J., Ridolfi, M., and Spang, R.: MIPAS level 2 operational analysis, Atmos. Chem. Phys., 6, 5605-5630, 2006, http://www.atmos-chem-phys.net/6/5605/2006/.

Remedios, J. J., Leigh, R. J., Waterfall, A. M., Moore, D. P., Sembhi, H., Parkes, I., Greenhough, J., Chipperfield, M. P., and Hauglustaine, D.: MIPAS reference atmospheres and comparisons to V4.61/V4.62 MIPAS level 2 geophysical data sets, Atmos. Chem. Phys. Discuss., 7, 9973-10017, 2007, http://www.atmos-chem-phys-discuss.net/7/9973/2007/.

Ridolfi, M., Carli, B., Carlotti, M., von Clarmann, T., Dinelli, B. M., Dudhia, A., Flaud, J.-M., Hoepfner, M., Morris, P. E., Raspollini, P., Stiller, G., and Wells, R. J.: Optimized forward model and retrieval scheme for MIPAS near-real-time data processing, Appl. Opt., 39(8), 1323-1340, 2000.
Ridolfi, M., Blum, U., Carli, B., Catoire, V., Ceccherini, S., Claude, H., De Clercq, C., Fricke, K. H., Friedl-Vallon, F., Iarlori, M., Keckhut, P., Kerridge, B., Lambert, J.-C., Meijer, Y. J., Mona, L., Oelhaf, H., Pappalardo, G., Pirre, M., Rizi, V., Robert, C., Swart, D., von Clarmann, T., Waterfall, A., and Wetzel, G.: Geophysical validation of temperature retrieved by the ESA processor from MIPAS/ENVISAT atmospheric limb-emission measurements, Atmos. Chem. Phys., 7, 4459-4487, 2007, http://www.atmos-chem-phys.net/7/4459/2007/.

Rodgers, C. D.: Inverse methods for atmospheric sounding: Theory and Practice, Series on Atmospheric, Oceanic and Planetary Physics, vol. 2, World Scientific Publishing Co., Singapore, 65-100, 2000

Simmons, A., Hortal, M., Untch, A., and Uppala, S.: Breakdown of the stratospheric winter polar vortex, ECMWF Newsl., 96, 2-10, 2003.

Spang, R., Remedios, J., and Barkley, M.: Colour indices for the detection and differentiation of cloud types in infrared limb emission spectra, Adv. Space Res., 33, 1041-1047, 2004.

Steck, T., Höpfner, M., von Clarmann, T., and Grabowski, U.: Tomographic retrieval of atmospheric parameters from infrared limb emission observations, Appl. Opt. 44, 3291-3301, 2005.

Stiller, G. P., von Clarmann, T., Funke, B., Glatthor, N., Hase, F., Höpfner, M., and Linden, A.: Sensitivity of trace gas abundances retrievals from infrared limb emission spectra to simplifying approximations in radiative transfer modeling, J. Quant. Spectrosc. Radiat. Transfer, 72, 249-280, 2002.

von Clarmann,T., Glatthor, N., Grabowski, U., Höpfner, M., Kellmann, S., Kiefer, M., Linden, A., Mengistu Tsidu, G., Milz, M., Steck, T., Stiller, G. P., Wang, D. Y., Fischer, H., Funke, B., Gil-López, S., and López-Puertas, M.: Retrieval of temperature and tangent altitude pointing from limb emission spectra recorded from space by the Michelson Interferometer for Passive Atmospheric Sounding (MIPAS), J. Geophys. Res. 108(D23), 4736, doi:10.1029/2003JD003602, 2003.

von Clarmann, T., Höpfner, M., Kellmann, S., Linden, A., Chauhan, S., Funke, B., Grabowski, U., Glatthor, N., Kiefer, M., Schieferdecker, T., Stiller, G. P., and Versick, S.: Retrieval of temperature, $\mathrm{H}_{2} \mathrm{O}, \mathrm{O}_{3}, \mathrm{HNO}_{3}, \mathrm{CH}_{4}, \mathrm{~N}_{2} \mathrm{O}, \mathrm{ClONO}_{2}$ and $\mathrm{ClO}$ from MIPAS reduced resolution nominal mode limb emission measurements, Atmos. Meas. Tech., 2, 159-175, 2009a, http://www.atmos-meas-tech.net/2/159/2009/.

von Clarmann, T., De Clercq, C., Ridolfi, M., Höpfner, M., and Lambert, J.-C.: The horizontal resolution of MIPAS, Atmos. Meas. Tech., 2, 47-54, 2009b, http://www.atmos-meas-tech.net/2/47/2009/.

Waters, W., Froidevaux, L., Harwood, R. S., at al.: The Earth Observing System Microwave Limb Sounder (EOS MLS) on the Aura satellite, IEEE Trans. Geosci. Remote Sens., 44, 1075-1092, 2006 